Article

\title{
Stability Analysis of an Eight Parameter SIR-Type Model Including Loss of Immunity, and Disease and Vaccination Fatalities
}

\author{
Florin Avram ${ }^{1, *} \mathbb{D}$, Rim Adenane ${ }^{2}$, Gianluca Bianchin ${ }^{3}$ and Andrei Halanay ${ }^{4}$ \\ 1 Laboratoire de Mathématiques Appliquées, Université de Pau, 64000 Pau, France \\ 2 Département des Mathématiques, Université Ibn-Tofail, Kenitra 14000, Morocco; rim.adenane@uit.ac.ma \\ 3 Department of Electrical, Computer, and Energy Engineering, University of Colorado Boulder, \\ Boulder, CO 80309, USA; gianluca.bianchin@colorado.edu \\ 4 Department of Mathematics and Informatics, Polytechnic University of Bucharest, 062203 Bucharest, \\ Romania; andrei.halanay@upb.ro \\ * Correspondence: Florin.Avram@univ-Pau.fr
}

check for updates

Citation: Avram, F.; Adenane, R.; Bianchin, G.; Halanay, A. Stability Analysis of an Eight Parameter SIR-Type Model Including Loss of Immunity, and Disease and Vaccination Fatalities. Mathematics 2022, 10, 402. https://doi.org/ $10.3390 /$ math10030402

Academic Editors: Mikhail Kolev and Dumitru Baleanu

Received: 16 December 2021

Accepted: 22 January 2022

Published: 27 January 2022

Publisher's Note: MDPI stays neutral with regard to jurisdictional claims in published maps and institutional affiliations.

Copyright: (c) 2022 by the authors. Licensee MDPI, Basel, Switzerland. This article is an open access article distributed under the terms and conditions of the Creative Commons Attribution (CC BY) license (https:// creativecommons.org/licenses/by/ $4.0 /)$.

\begin{abstract}
We revisit here a landmark five-parameter SIR-type model, which is maybe the simplest example where a complete picture of all cases, including non-trivial bistability behavior, may be obtained using simple tools. We also generalize it by adding essential vaccination and vaccinationinduced death parameters, with the aim of revealing the role of vaccination and its possible failure. The main result is Theorem 1, which describes the stability behavior of our model in all possible cases.
\end{abstract}

Keywords: epidemic models; varying population models; stability; next-generation matrix approach; basic reproduction number; vaccination; loss of immunity; endemic equilibria; isoclines

\section{Introduction}

Motivation. This paper has the dual purpose of providing a short guide to students of deterministic mathematical epidemiology, among which we count ourselves, and to simultaneously illustrate the technical work one is faced with in an elementary, but not simple "exercise". Of course, one can easily find at least five must-read excellent textbooks and theses surveying this field (with different emphases, e.g., epidemiological, stability, or control); see, for example, the Refs. [1-8], and also at least a hundred major papers which are a must-read. We hope, however, that our little guide may help future students decide the order in which these materials must be assimilated.

A bit of history. Deterministic mathematical modelling of diseases started with the works of Ross on malaria, and imposed itself after the work of the Ref. [9] on the Bombay plague of 1905-1906. This was subsequently followed by works on measles, smallpox, chickenpox, mumps, typhoid fever, and diphtheria (see, for example, [10]), and recently, the COVID-19 pandemic (see, for example, the Refs. [11-23], to cite just a few examples within the wide range of literature). Note that at its beginning, mathematical epidemiology was a collection of similar examples dealing with current epidemics (the first precise mathematical framework for the field which we are aware of is in the recent paper, [24]).

The literature on deterministic mathematical epidemiology may be divided into three streams.

1. "Constant total population" models are the easiest to study. However, since death is an essential factor of epidemics, the assumption of a constant total population (clearly a short-term or very large population approximation) deserves some comment. One possible rigorous justification for deterministic constant population epidemiological models comes from slow-fast analysis [25-27]. This is best understood for models 
with demography (birth, death), which typically happen on a slower scale than the infectious phenomena. Here there is a natural partition of the compartments into a vector $\vec{i}(t) \in \mathbb{R}_{+}^{-}$of disease/infectious compartments (asymptomatic, infectious, hospitalized, etc). These interact (quickly) with the other input classes, such as the susceptibles, and output classes, such as the recovered and dead.

For "constant total population" models, the total population $N$ clearly plays no role, and one may use the assumption that the rate of infection is independent of $N$, of the form $\beta S(t) I(t)$ (which was called pseudo, or simple mass-action incidence [28]). Note that this simplification of the rate of infection (adopted in the majority of the literature) is inappropriate for varying population models.

2. Models with a constant birth rate (in the analog stochastic model, this would correspond to emigration). These models include the previous class, and preserve some of its nice features, like the uniqueness of the endemic fixed point. They typically satisfy the " $\mathcal{R}_{0}$ alternative", established via the next-generation matrix approach, and also the fact that the endemic point exists only when $\mathcal{R}_{0}>1$-see [29] for a recent and well-written review of several stability results for these classes of models.

3. Finally, we arrive at the class our paper is concerned with-models with linear birth rates, $\Lambda N$ (or a constant birth rate per capita in the analog stochastic model), varying total population $N$, and a "proportionate mixing" rate of infection:

$$
\beta S(t) \frac{I(t)}{N(t)}
$$

As far as we know, this stream of literature was initiated by the Refs. [30,31], and allows the possibility of bi-stability when $\mathcal{R}_{0}<1$ (absent from the previous models), even in a SIR example with five parameters [32]. (This reveals that for an initially high number of infectives, the trajectory may lie in the basin of attraction of a stable endemic point instead of being eradicated. The discrepancy with what is expected from the corresponding stochastic model suggests that the deterministic model is inappropriate in this range.)

This last stream of literature is quite important, since a constant birth rate per capita is a natural assumption.

Despite further remarkable works on particular examples-see, for example, the Refs. [33-39] (which preferred all direct stability analyses to the next-generation matrix approach) - the literature on models with varying total populations, unlike the two preceding streams, has not yet reached a general result.

To understand this failure, it seemed to us a good idea to revisit an important SIR model with disease-induced deaths and loss of immunity [32,35]. These important works already illustrate some of the complexities which may arise for varying population models, particularly the possibility of bi-stability when $\mathcal{R}_{0}<1$. This surprising fact led us to introduce the concept of strong global stability of the DFE (disease-free Equilibrium), and to find conditions for this to hold in our example (see Proposition 4). Since the method used is simply linear programming, we hope to extend this in future work.

Our model. We further added to the extension of the Ref. [9] introduced in vaccinations of the Ref. [32,35]. Grouping together the recovered and vaccinated, our "SIR/V+S" model is described by: 


$$
\begin{aligned}
S^{\prime}(t) & =\Lambda N(t)-\frac{\beta}{N(t)} S(t) I(t)-\left(\gamma_{s}+\mu\right) S(t)+\gamma_{r} R(t), \\
I^{\prime}(t) & =I(t)\left(\frac{\beta}{N(t)} S(t)+\frac{\beta_{r}}{N(t)} R(t)-\gamma-\mu-v_{i}\right), \\
R^{\prime}(t) & =\gamma_{s} S(t)+\gamma I(t)-\left(\gamma_{r}+\mu+v_{r}\right) R(t)-\frac{\beta_{r}}{N(t)} R(t) I(t), \\
D^{\prime}(t) & =\mu(S(t)+I(t)+R(t)), \\
D_{e}^{\prime}(t) & =v_{i} I(t)+v_{r} R(t), \\
N^{\prime}(t) & =(S(t)+I(t)+R(t))^{\prime}=(\Lambda-\mu) N(t)-v_{i} I(t)-v_{r} R(t) .
\end{aligned}
$$

It involves six states: $S: \mathbb{R}_{>0} \rightarrow \mathbb{N}_{>0}$ describing the number of susceptible individuals in the population, $I: \mathbb{R}_{\geq 0} \rightarrow \mathbb{N}_{>0}$ describing the number of infections, $R: \mathbb{R}_{\geq 0} \rightarrow \mathbb{N}_{>0}$ describing the number of recovered or vaccinated, $D: \mathbb{R}_{\geq 0} \rightarrow \mathbb{N}_{>0}$ describing the number of natural deaths in the population, $D_{e}: \mathbb{R}_{\geq 0} \rightarrow \mathbb{N}_{>0}$ describing the number of deaths originated by the disease, and $N: \mathbb{R}_{\geq 0} \rightarrow \mathbb{N}_{>0}$ describing the total number of (alive) individuals in the population.

The parameters $\Lambda \in \mathbb{R}_{\geq 0}$ and $\mu \in \mathbb{R}_{\geq 0}$ denote the average birth and death rates in the population (in the absence of the disease), respectively, $\gamma_{s} \in \mathbb{R}_{\geq 0}$ is the vaccination rate, $\gamma_{r} \in \mathbb{R}_{\geq 0}$ denotes the rate at which immune individuals lose immunity (this is the reciprocal of the expected duration of immunity), $\gamma \in \mathbb{R}_{>0}$ is the rate at which infected individuals recover from the disease, $v_{i} \in \mathbb{R}_{\geq 0}$ is the extra death rate due to the disease, and $v_{r} \in \mathbb{R}_{\geq 0}$ is the average death rate in the recovered/vaccinated compartment (due to, e.g., deaths caused by the vaccine). Note that in what follows, we use the notation $\gamma_{c}$ to denote the total rate at which individuals leave a certain compartment $C$ towards other non-deceased compartments, and we use $v_{c}$ to denote the rate at which individuals leave compartment $C$ towards a deceased compartment.

In (1), susceptible individuals become infected at rate $\frac{\beta}{N(t)} I(t)$ (thus moving to the $I$ compartment), they are vaccinated at rate $\gamma_{s}$ (thus moving to the $R$ compartment), and deaths occur at rate $\mu$ (thus moving to the $D$ compartment); infected individuals recover at rate $\gamma$ (thus moving to the $R$ compartment), die of non-disease related causes at rate $\mu$ (thus moving to the $D$ compartment), and die of disease-related causes at rate $v_{i}$ (thus moving to the $D_{e}$ compartment); recovered individuals lose immunity at rate $\gamma_{r}$ (thus moving to the $S$ compartment), die of non-disease-related causes at rate $\mu$ (thus moving to the $D$ compartment), die of disease-related causes at rate $v_{r}$, and become re-infected at rate $\frac{\beta_{r}}{N(t)} I(t)$ (thus moving to the $I$ compartment) (see Remark 1 for a discussion on re-infections).

Note that $D, D_{e}$ are completely determined once the other classes are found. These "output classes" will not be mentioned further (since they are only relevant in control problems, which are outside our scope). The dynamics of $I$, the disease class, allows to compute the basic reproduction number via the next-generation matrix method. Finally, the input classes $S, R$ determine the disease-free Equilibriumby themselves. We will analyze the research content based on Figures 1-6. 


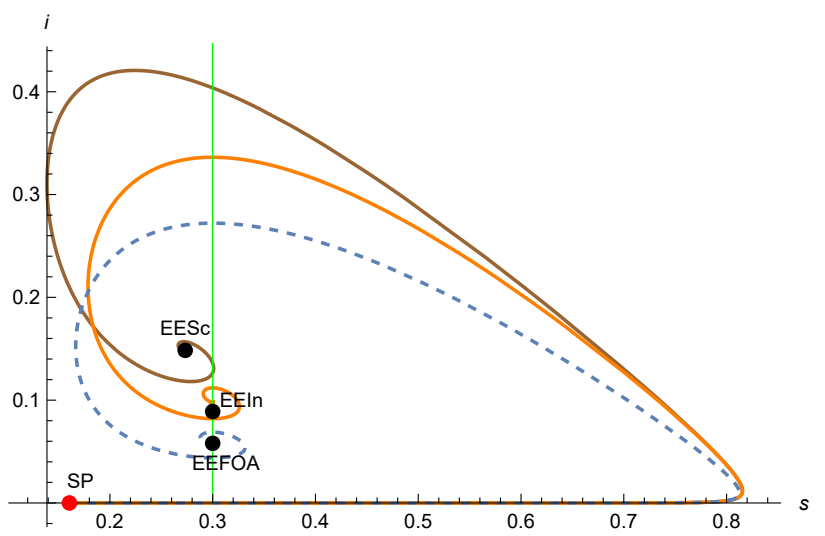

- Scaled epidemic model

Intermediate approximation

Figure 1. Parametric (s,i) plots of the scaled epidemic (9) and its FA (12) and intermediate approximations for a SIR-type model with one infectious class, starting from a starting point SP with $i_{0}=10^{-6}, \mathcal{R}=3.21$, critical vaccination 0.622222 , and $\gamma_{s}=0.01$. The other parameters are $\beta=5, \gamma=1 / 2, \Lambda=\mu=1 / 10, \gamma_{r}=1 / 6, v_{i}=0.9, v_{r}=0$. EESc, EEIn,EEFOA are the stable endemic points of the scaled model, intermediate model, and the FA model, respectively. The green vertical line denotes the immunity threshold $1 / \mathcal{R}=s_{E E F O A}=s_{E E I n}$. Note that the epidemic will at first spend a long time (since births and deaths have slow rates as compared to the disease) in the vicinity of the manifold $\vec{i}(t)=0$, where the three processes are indistinguishable, before turning towards the endemic equilibrium point(s).

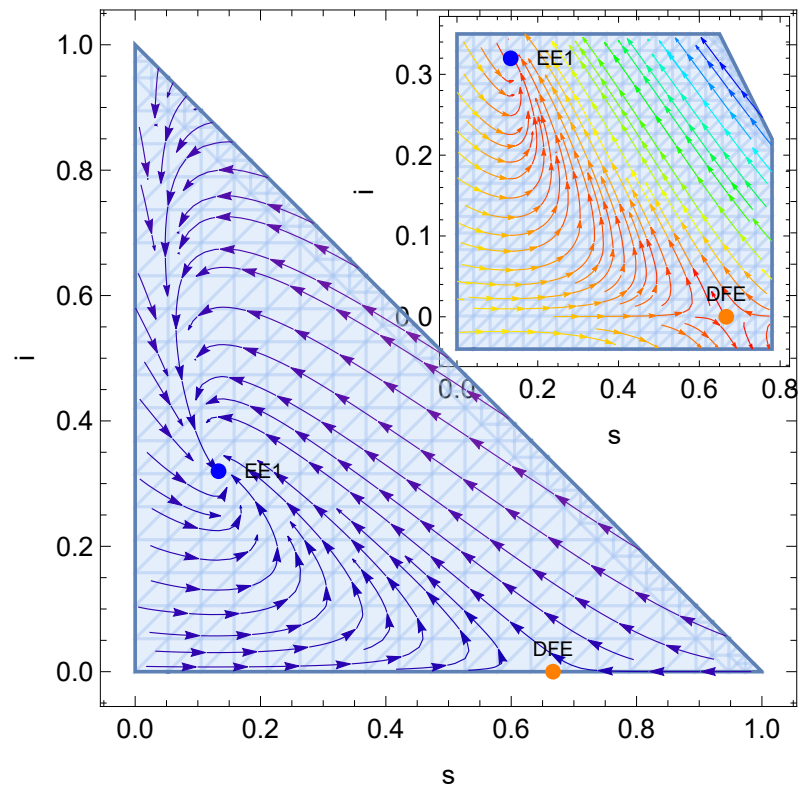

Figure 2. Stream plots of $(s, i)$ for the scaled model (13) illustrating the case when $\gamma_{s}$ is less than the critical vaccination $\gamma_{s}^{*}$ defined in (24), EE1 is a stable equilibrium point, DFE is a (boundary) saddle point, and EE2 is outside the domain. 


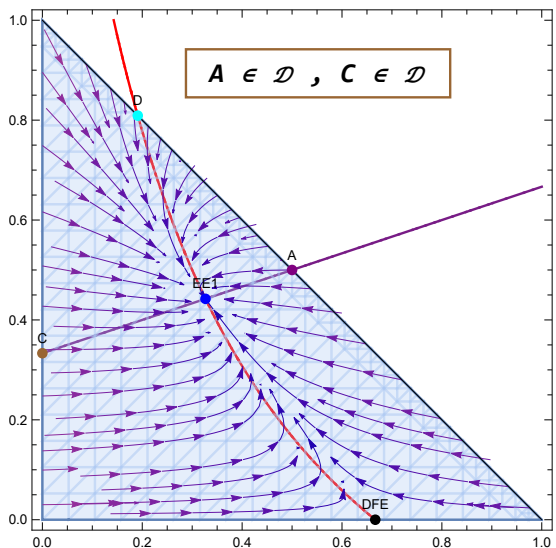

(a)

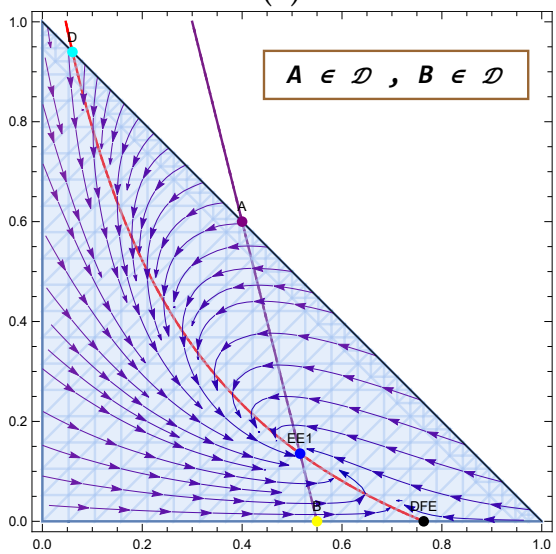

(c)

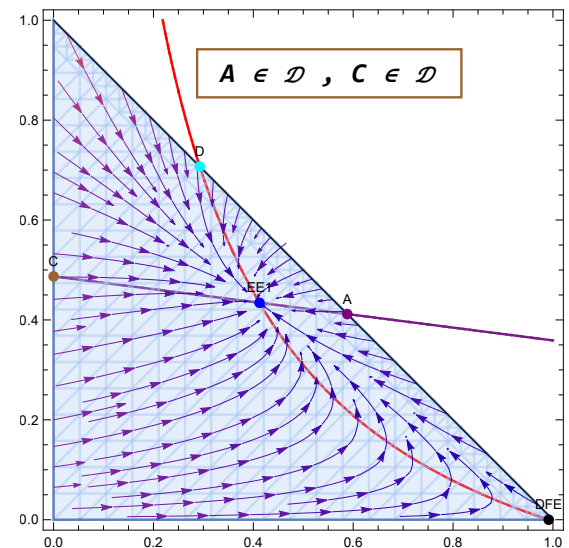

(b)

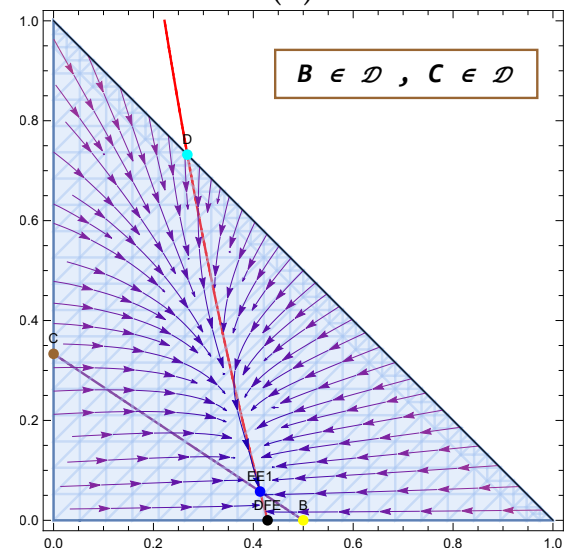

(d)

Figure 3. Stream plots of $(s, i)$ when $\gamma_{s}>0$, in four cases when $\mathcal{R}_{0}>1$. The DFE is a saddle point and EE is a stable sink. (a) $\beta=5>\beta_{r}=4>3=\Lambda+\gamma+v_{i}=1+1+1, \gamma_{r}=1, \gamma_{s}=1>\gamma_{s}^{*} \Leftrightarrow s_{B}>$ $1, \beta>\beta_{r}>\gamma+v_{i}+\Lambda$. (b) $\beta_{r}=4>\beta=3.5>0.21=\Lambda+\gamma+v_{i}=1+1+0.1, \gamma_{r}=1 / 6, \gamma_{s}=0.01$. (c) $B, A \in \mathcal{D}$, with $\beta=0.3>0.21=\Lambda+\gamma+v_{i}=0.01+0.05+0.15>\beta_{r}=0.1, \gamma_{r}=1 / 26, \gamma_{s} .=$ $0.015<\gamma_{s}^{*} \Leftrightarrow s_{B}<s_{d f e}$. (d) $B, C \in \mathcal{D}$, with $\mathcal{R}_{0}=1.04762>1, \beta=2<\Lambda+\gamma+v_{i}=1+1+1=$ $3<\beta_{r}=4, \gamma_{r}=1 / 2, \gamma_{s}=2>\gamma_{s}^{*} \Leftrightarrow s_{B}>s_{d f e}$.

Remark 1. Models of the form (1) that account for non-constant population sizes can be especially useful in two scenarios: (i) to study diseases that remain infectious for long periods of time with a small disease mortality rate, where the natural death/birth rate of the population plays a central role (such as HIV/AIDS, malaria and tuberculosis), as well as (ii) to study diseases with short infectious periods but with a substantial disease mortality rate, where the death rate due to the disease plays a central role (such as measles, influenza, SARS/COVID).

The two-way transfers between the recovered and infected compartments (recall that $R \underset{\gamma}{\stackrel{\beta_{r}}{\rightleftarrows}} I$ ). can be used to account for multiple variants of the disease, whereby immunity to one variant does not guarantee immunity to all other variants. For instance, diseases such as HIV can develop resistance to medications, and such resistance can be transmitted to a partner. In these cases, even when the second party has recovered, it may become re-infected with a different variant. Notice that an even more general case is considered in the Ref. [35], where vaccinated individuals may transition to the infected compartment. 


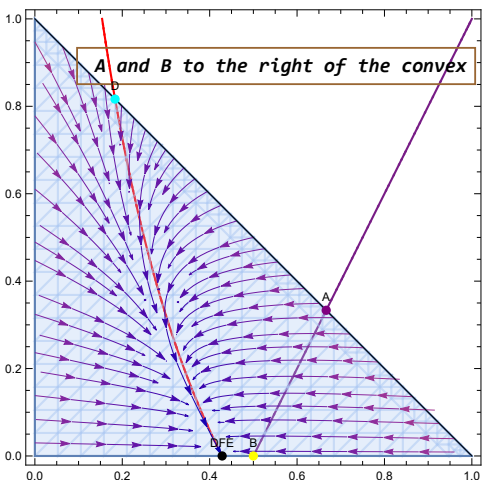

(a)

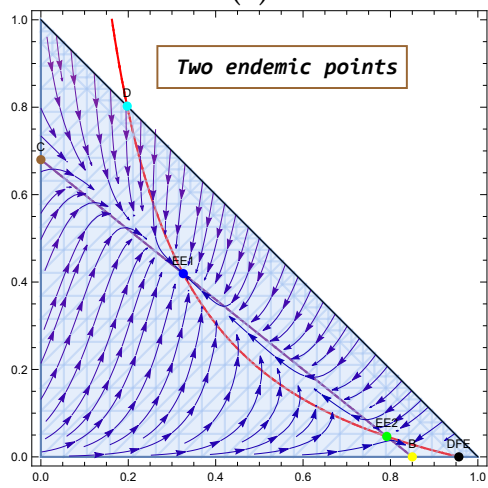

(d)

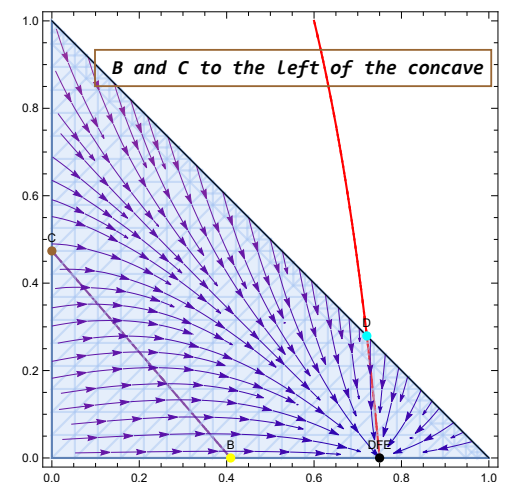

(b)

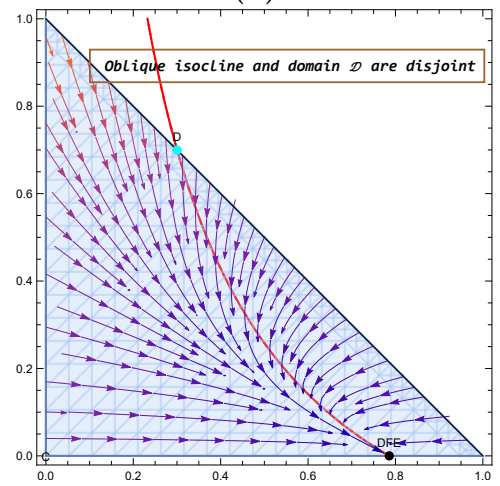

(e)

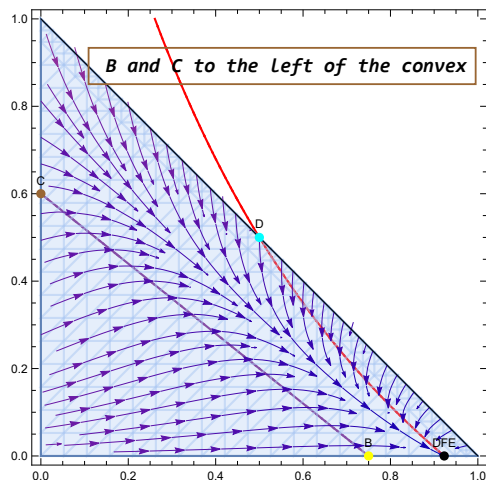

(c)

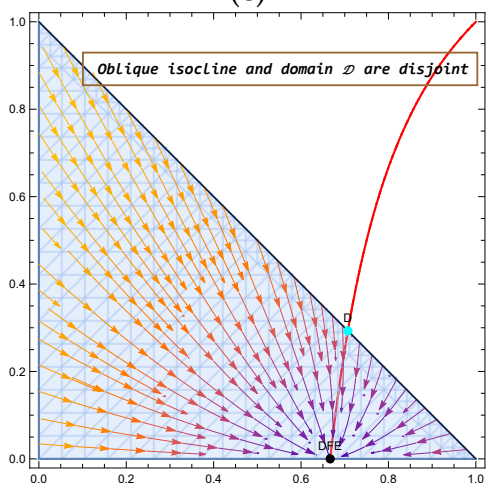

(f)

Figure 4. Stream plots of $(\mathrm{s}, \mathrm{i})$ when $\gamma_{s}>0$, in six cases when $\mathcal{R}_{0}<1$. The DFE is the only stable sink in the boundary of the domain, with the exception of case $(d)$, when EE1 $=(0.313823,0.428942)$ is a second sink, and EE2 $=(0.799177,0.0406582)$ is a saddle point. (a) $\mathcal{R}_{0}=0.952381<1, \beta=$ $4, \beta_{r}=2, \Lambda+\gamma+v_{i}=1+1+1=3, \beta_{r}^{(+)}=1.625, \gamma_{r}=1 / 2, \gamma_{s}=2, \beta_{r}<\Lambda+v_{i}+\gamma<\beta, v_{i}<$ $\beta, \beta_{r}>\beta_{r}^{(+)}$, and $\gamma_{s}>\gamma_{s}^{*} \Leftrightarrow s_{B}>s_{d f e}$. (b) $\beta=0.03, \beta_{r}=0.25, \Lambda+\gamma+v_{i}=0.03+0.07+0.06=$ $0.16, \beta_{r}^{(+)}=0.0219597, \gamma_{r}=0.03, \gamma_{s}=0,02, \beta<v_{i}<\Lambda+v_{i}+\gamma<\beta_{r}, \beta_{r}>\beta_{r}^{(+)}$, and $\gamma_{s}<\gamma_{s}^{*} \Leftrightarrow$ $s_{B}<s_{d f e}$. (c) $\beta=0.1, \beta_{r}=0.3, \Lambda+\gamma+v_{i}=0.03+0.07+0.05=0.15, \beta_{r}^{(+)}=0.729883, \gamma_{r}=$ $0.03, \gamma_{s}=0,02, v_{i}<\beta<\Lambda+v_{i}+\gamma \leq \beta_{r}, \beta_{r}<\beta_{r}^{(+)}$, and $\gamma_{s}<\gamma_{s}^{*} \Leftrightarrow s_{B}<s_{d f e}$. (d) $\beta=0.2, \beta_{r}=$ $0.4, \Lambda+\gamma+v_{i}=0.01+0.07+0.15=0.23, \beta_{r}^{(+)}=0.327443, \gamma_{r}=10^{-4}, \gamma_{s}=0,002, v_{i}<\beta<$ $\Lambda+\gamma+v_{i}<\beta_{r}, \beta_{r} \geq \beta_{r}^{(+)}$, and $s_{B}<s_{d f e}$. (e) $\Lambda+\gamma+v_{i}=1+2.1+0.1=3.2>\beta_{r}=3.1>\beta=$ $3, \gamma_{r}=0.1, \gamma_{s}=0.3, \beta<\beta_{r}<\Lambda+\gamma+v_{i}$, and $\beta>v_{i}$. (f) $\Lambda+\gamma+v_{i}=1+1+2.5=4.5>\beta=$ $0.5>\beta_{r}=0.25, \gamma_{r}=1, \gamma_{s}=1, \beta_{r}<\beta<\Lambda+\gamma+v_{i}$, and $\beta<v_{i}$.

Remark 2. In practical situations, certain parameter relations, for instance, $\beta_{r}>\beta, v_{r}>v_{i}$, might seem "unreasonable from a medical point of view". In what follows, we choose not to assume any relationship among the parameters in (1) in order to highlight the fact that surprising mathematical behaviors, such as bistability—see Figure 4d-may arise when "things go wrong".

We now make several remarks which serve as an appetizer for the rest of the paper.

Remark 3. The critical value $\beta=\beta_{r}$ defines a model where both the sickness and the vaccination do not affect the infectivity (neither confers any immunity). The recovered class might be better 
viewed, then, as a susceptible class $S_{o}$ of older individuals, with extra mortality rate $v_{r}>0$ and no births:

$$
\begin{aligned}
& S^{\prime}(t)=\Lambda N(t)-\frac{\beta}{N(t)} S(t) I(t)-\left(\gamma_{s}+\mu\right) S(t)+\gamma_{r} S_{o}(t), \\
& S_{o}^{\prime}(t)=-\frac{\beta}{N(t)} S_{o}(t) I(t)-\left(\gamma_{r}+\mu+v_{r}\right) S_{o}(t)+\gamma_{s} S(t)+\gamma I(t), \\
& I^{\prime}(t)=I(t)\left(\frac{\beta}{N(t)}\left(S(t)+S_{o}(t)\right)-\gamma-\mu-v_{i}\right) .
\end{aligned}
$$

A moment of reflection reveals that this particular case has two surprising features: (a) after the infection is over, transfers only occur towards the old class, $S_{0}$, and $(b)$ transfers between the two age groups occur; it is hard to make sense of this without further imposing $\gamma_{s}=\gamma_{r}=0$.

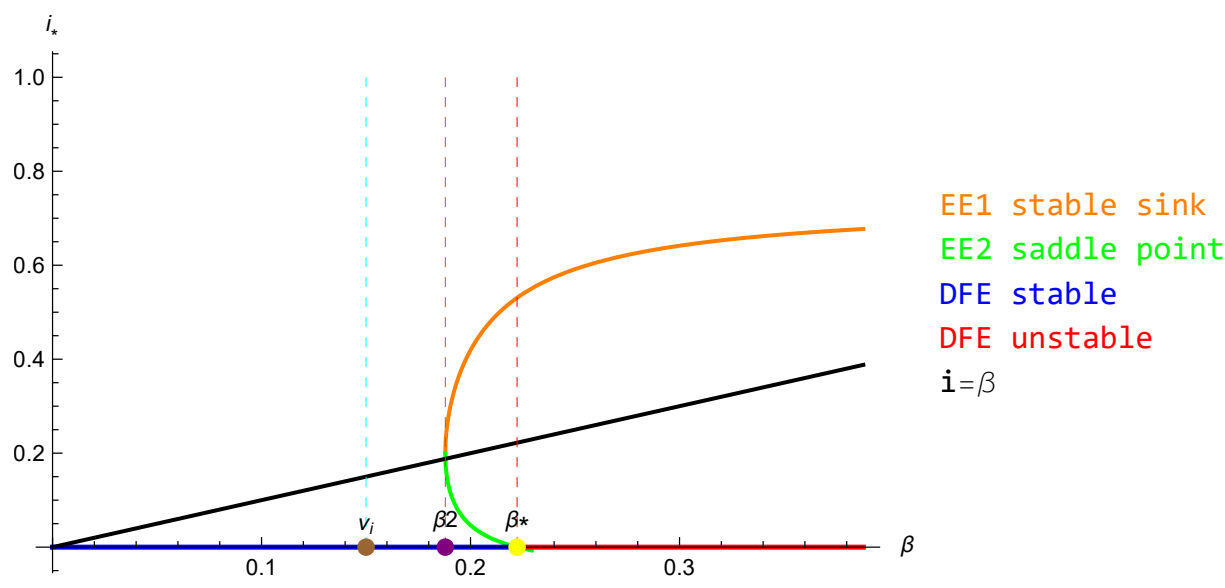

Figure 5. Diagram bifurcation with respect to $\beta$, in the case $\max \left[\Lambda+\gamma+v_{i}, \beta_{r}^{(+)}\right]<\beta_{r}$. For $\beta<v_{i}$ we are in the case of Figure $4 \mathrm{~b}$ (Theorem 1(2(b))), with the immunity line to the left of the hyperbola, and no endemic points. The same situation occurs for $v_{i}<\beta<\beta_{2}$, where $\beta_{2}$ is the largest root of $\Delta(\beta)=0$, except that the hyperbola changes to convex-see Figure 4c (Theorem 1(2(c)(i))). After $\beta_{2}$, two endemic points emerge-see Figure $4 \mathrm{~d}$ (Theorem 1(2(c)(ii))). The lower endemic point exits through the boundary $i=0$ at $\beta^{*}$, defined in (31), after which the remaining endemic point remains the only stable point.
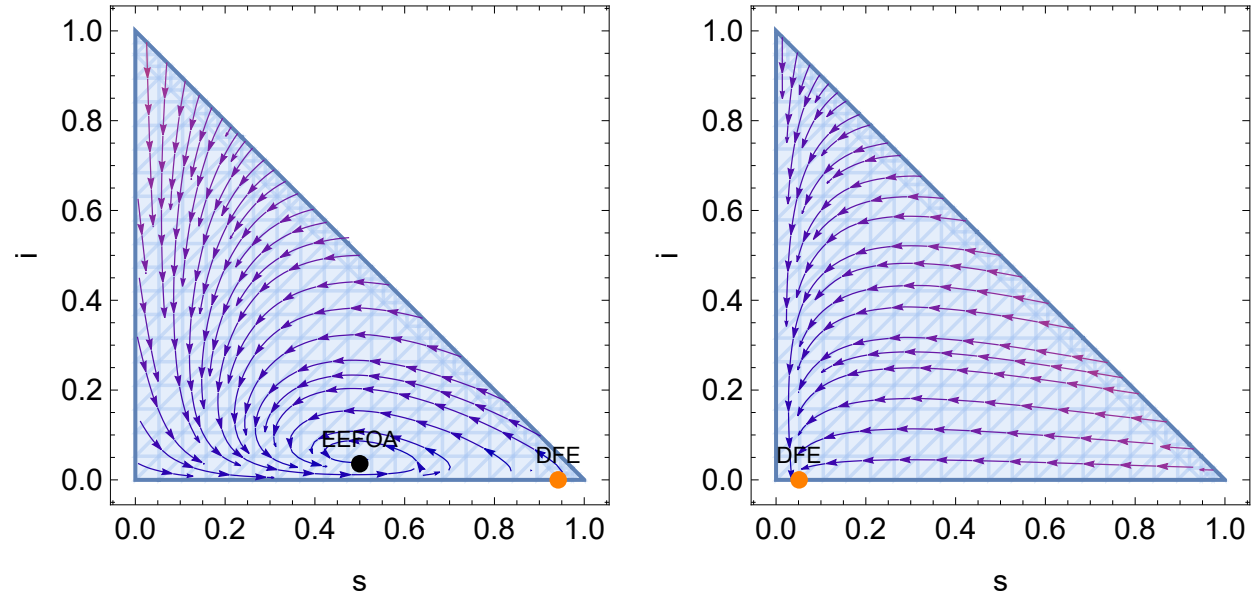

Figure 6. Stream plots of $(s, i)$ for the FA model when $\gamma_{s} \in\{1 / 100,3\}$ is smaller and bigger, respectively, than the critical vaccination $\gamma_{s}^{*}=0.239087$ defined in (25).

Remark 4. When $\beta_{r}=0$, our model is an example of the matrix-SIR Arino model with a linear birth rate, a class of models for which only few general results are available [40]. 
In what follows, we will investigate the stability properties of an equivalent system to (1) obtained by investigating the normalized quantities $\mathrm{s}=\frac{S}{N}, \mathrm{i}=\frac{I}{N}, \mathrm{r}=\frac{R}{N}$ and given by (see Proposition 1, Section 2 for a detailed derivation).

$$
\left\{\begin{array}{l}
\mathrm{s}^{\prime}(t)=\Lambda+\gamma_{r} \mathrm{r}(t)-\left(\beta \mathrm{i}(t)+\gamma_{s}\right) \mathrm{s}(t)+\mathrm{s}(t)\left(-\Lambda+v_{i} \mathrm{i}(t)+v_{r} \mathrm{r}(t)\right) \\
\mathrm{i}^{\prime}(t)=\mathrm{i}(t)\left[\beta \mathrm{s}(t)+v_{i} \mathrm{i}(t)+\left(v_{r}+\beta_{r}\right) \mathrm{r}(t)-\left(\gamma+v_{i}+\Lambda\right)\right] \\
\mathrm{r}^{\prime}(t)=\gamma \mathrm{i}(t)+\gamma_{s} \mathrm{~s}(t)-\left(\gamma_{r}+\Lambda+v_{r}\right) \mathrm{r}(t)+\mathrm{r}(t)\left(-\beta_{r} \mathrm{i}(t)+v_{i} \mathrm{i}(t)+v_{r} \mathrm{r}(t)\right) \\
\mathrm{s}(t)+\mathrm{i}(t)+\mathrm{r}(t)=1
\end{array}\right.
$$

Remark 5. Note that this reduces to the classic SIR model [9] when $\Lambda=v_{i}=v_{r}=\gamma_{r}=\gamma_{s}=$ $\beta_{r}=0$.

Remark 6. Factoring the second equation reveals the threshold after which the infection starts decreasing:

$$
\beta \mathrm{s}(t)+v_{i} \mathrm{i}(t)+\left(v_{r}+\beta_{r}\right) r(t)<\Lambda+\gamma+v_{i}
$$

herd immunity $(\mathrm{s}, \mathrm{i})$ line, or max-line (line since $\mathrm{r}=1-\mathrm{s}-\mathrm{i})$. Notice that, in contrast with the case of models with a constant population size and no loss of immunity where the herd immunity condition depends only on the susceptible state, the above condition depends on three states $(\mathrm{s}, \mathrm{i}, \mathrm{r})$. Additionally, when $v_{i}=v_{r}=\beta_{r}=0$, this reduces to the well-studied herd immunity threshold.

(1) The inequality obtained at the DFE, when $\mathrm{i}=0$,

$$
\beta s_{d f e}+\left(v_{r}+\beta_{r}\right) r_{d f e} \leq \Lambda+\gamma+v_{i} \Leftrightarrow \frac{\beta s_{d f e}+\left(v_{r}+\beta_{r}\right) r_{d f e}}{\Lambda+\gamma+v_{i}}:=\mathcal{R}_{0}<1
$$

turns out to ensure the local stability of the disease-free equilibrium (disease-free Equilibrium)-see Section 3.1.

(2) $\mathcal{R}_{0}$ introduced above coincides with the famous basic reproduction number defined via the next-generation matrix approach.

(3) The disease-free equilibrium (obtained by plugging $\mathrm{i}=0, \mathrm{r}=1-\mathrm{s}$ in the fixed point equation) is such that

$$
s_{d f e}:=\frac{\Lambda+\gamma_{r}}{\Lambda+\gamma_{r}+\gamma_{s}} \in[0,1], \quad s_{d f e}=1 \Leftrightarrow \gamma_{s}=0
$$

Remark 7. (A) The equality $\mathcal{R}_{0}=1$ is linear in $\gamma_{s}$ and yields the so-called "critical vaccination"

$$
\gamma_{s}^{*}=\left(\Lambda+\gamma_{r}\right) \frac{\beta-\left(\Lambda+\gamma+v_{i}\right)}{\Lambda+\gamma+v_{i}-\beta_{r}}
$$

provided that the denominator does not blow-up.

This formula is positive if either $\beta_{r} \leq \Lambda+\gamma+v_{i} \leq \beta$, or $\beta \leq \Lambda+\gamma+v_{i} \leq \beta_{r}$. When $\beta_{r}=0$, we recover a classical critical vaccination formula

$$
\gamma_{s}^{*}=\left(\Lambda+\gamma_{r}\right)(\mathcal{R}-1), \mathcal{R}=\frac{\beta}{\Lambda+\gamma+v_{i}} .
$$

(B) The equality $\mathcal{R}_{0}=1$ is also linear in $\beta$ and yields a "critical contact rate"

$$
\beta^{*}=\frac{\left(\gamma+v_{i}+\Lambda\right)\left(\Lambda+\gamma_{r}+\gamma_{s}\right)-\beta_{r} \gamma_{s}}{\Lambda+\gamma_{r}}
$$

It may be checked that at this critical value, the value $i_{e e}$ of the infectious at the lower endemic point crosses the $i=0$ axis, and may reduce the number of endemic points from 3 to 2 -see Figure 5 for details. 
Contents. Section 2 reviews the dimension reduction available for the proportions of models with a linear birth rate, and emphasizes the fact that the well-studied deterministic model is an approximation of the model with a linear birth rate studied here. A second, finer "intermediate approximation" is introduced as well.

Section 3.1 computes $\mathcal{R}_{0}$ via the next-generation matrix approach (NGM), thereby establishing the well-known weak $\mathcal{R}_{0}$ alternative [41]; it also introduces the concept of "strong global stability" of the DFE in Proposition 4, which may be useful for more advanced models.

The endemic equilibria are discussed in Section 4.

Section 5 identifies more precisely, in the particular case $v_{r}=0$, the case when global stability does not hold. The results heavily involve the vaccination parameter $\gamma_{s}$ and its critical value. The increased complexity of the model forces a geometric approach, already hinted at in [Section 4] of [32]. This ends up in the consideration of 10 cases, one of which, Theorem 1(2(c)), remains only partly resolved.

Section 6 discusses the particular case $\gamma_{s}=0$, generalizing and providing missing details of the results in [Section 4] of [32].

Section 7 gives simpler results for the first approximation FA (actually for a slightly more general "classic/pedagogical model").

Finally, Section A provides the proof of a technical result, and Section 8 reviews the pillar of deterministic epidemic models: the definition of the basic reproduction number via the next-generation matrix method.

\section{Dimension Reduction for the SIR/V+S Model with Linear Birth Rate}

It is convenient to reformulate (1) in terms of the normalized fractions

$$
\mathrm{s}=\frac{S}{N}, \quad \mathrm{i}=\frac{I}{N}, \quad \mathrm{r}=\frac{R}{N} .
$$

This process, sometimes called "non-dimensionalizing" (see for example [42]), allows us to provide the following equivalent representation of (1).

Proposition 1. Let $\mathrm{s}, \mathrm{i}, \mathrm{r}$ be as defined in (8). Then, the dynamics (1) can be equivalently rewritten as:

$$
\begin{aligned}
& \mathrm{s}^{\prime}(t)=\Lambda+\gamma_{r} \mathrm{r}(t)-\left(\beta \mathrm{i}(t)+\gamma_{s}\right) \mathrm{s}(t)+\mathrm{s}(t)\left(-\Lambda+v_{i} \mathrm{i}(t)+v_{r} \mathrm{r}(t)\right), \\
& \mathrm{i}^{\prime}(t)=\mathrm{i}(t)\left[\beta \mathrm{s}(t)+v_{i} \mathrm{i}(t)+\left(v_{r}+\beta_{r}\right) \mathrm{r}(t)-\left(\gamma+v_{i}+\Lambda\right)\right], \\
& \mathrm{r}^{\prime}(t)=\gamma \mathrm{i}(t)+\gamma_{s} \mathrm{~s}(t)-\left(\gamma_{r}+\Lambda+v_{r}\right) \mathrm{r}(t)+\mathrm{r}(t)\left(-\beta_{r} \mathrm{i}(t)+v_{i} \mathrm{i}(t)+v_{r} \mathrm{r}(t)\right), \\
& \mathrm{s}(t)+\mathrm{i}(t)+\mathrm{r}(t)=1 .
\end{aligned}
$$

Proof. By using

$$
N^{\prime}(t) / N(t)=(\Lambda-\mu)-v_{i} \mathrm{i}(t)-v_{r} \mathrm{r}(t),
$$

we obtain for the susceptibles:

$$
\begin{aligned}
\mathrm{s}^{\prime}(t) & =\frac{S^{\prime}}{N}-\frac{N^{\prime}}{N^{2}} S \\
& =\frac{\Lambda(N)}{N}-\frac{\beta S(t) I(t)}{N^{2}}+\frac{\gamma_{r} R(t)-\left(\mu+\gamma_{s}\right) S(t)}{N}-s(t) \frac{(\Lambda-\mu)-v_{i} \mathrm{i}(t)-v_{r} \mathrm{r}(t)}{N} \\
& =\Lambda(1-\mathrm{s}(t))-\beta \mathrm{s}(t) \mathrm{i}(t)+\gamma_{r} \mathrm{r}(t)+\mathrm{s}(t)\left(\mu+v_{i} \mathrm{i}(t)+v_{r} \mathrm{r}(t)-\mu-\gamma_{s}\right) \\
& =\Lambda-\beta \mathrm{s}(t) \mathrm{i}(t)+\gamma_{r} \mathrm{r}(t)-\left(\gamma_{s}+\Lambda\right) \mathrm{s}(t)+\mathrm{s}(t)\left(v_{i} \mathrm{i}(t)+v_{r} \mathrm{r}(t)\right) .
\end{aligned}
$$


Similarly,

$$
\begin{aligned}
\mathrm{i}^{\prime}(t) & =\frac{I^{\prime}}{N}-\frac{N^{\prime}}{N^{2}} I=\beta \mathrm{s}(t) \mathrm{i}(t)-\left(\gamma+\mu+v_{i}\right) \mathrm{i}(t)-I(t) \frac{(\Lambda-\mu)-v_{i} \mathrm{i}(t)-v_{r} \mathrm{r}(t)}{N} \\
& =-\mathrm{i}(t) \Lambda+\mathrm{i}(t)\left(\beta \mathrm{s}(t)+\beta_{r} \mathrm{r}(t)\right)-\left(\gamma+\mu+v_{i}\right) \mathrm{i}(t)+\mathrm{i}(t)\left(\mu+v_{i} \mathrm{i}(t)+v_{r} \mathrm{r}(t)\right) \\
& =\mathrm{i}(t)\left[\beta \mathrm{s}(t)+v_{i} \mathrm{i}(t)+\left(\beta_{r}+v_{r}\right) \mathrm{r}(t)-\left(\Lambda+\gamma+v_{i}\right)\right],
\end{aligned}
$$

and

$$
\begin{aligned}
\mathrm{r}^{\prime}(t) & =\frac{R^{\prime}}{N}-\frac{N^{\prime}}{N^{2}} R \\
& =\gamma \mathrm{i}(t)+\gamma_{s} \mathrm{~s}(t)-\left(\gamma_{r}+\mu+v_{r}\right) \mathrm{r}(t)-R(t) \frac{(\Lambda-\mu)-v_{i} \mathrm{i}(t)-v_{r} \mathrm{r}(t)}{N} \\
& =\gamma \mathrm{i}(t)+\gamma_{s} \mathrm{~s}(t)-\left(\gamma_{r}+\Lambda+v_{r}\right) \mathrm{r}(t)+\mathrm{r}(t)\left(-\beta_{r} \mathrm{i}(t)+v_{i} \mathrm{i}(t)+v_{r} \mathrm{r}(t)\right) .
\end{aligned}
$$

Finally, $\mathrm{s}(t)+\mathrm{i}(t)+\mathrm{r}(t)=1$ follows from $N^{\prime}(t)=(S(t)+I(t)+R(t))^{\prime}$ by substituting (8), which proves the equivalence between (1) and (9).

Remark 8. Note that the natural death rate $\mu$ does not intervene in (9), which is to be expected. Indeed, since this rate is the same for all the compartments, it has no effect on the fractions.

Remark 9. Note that the conservation equation

$$
\mathrm{n}:=\mathrm{s}+\mathrm{i}+\mathrm{r}=1 \text {, }
$$

in general, does not follow from the first three equations in (9). Indeed, by summing up the right-hand sides, we have:

$$
\mathrm{n}^{\prime}(t)=[1-\mathrm{s}(t)-\mathrm{r}(t)-\mathrm{i}(t)]\left(\Lambda-v_{i} \mathrm{i}(t)-v_{r} \mathrm{r}(t)\right),
$$

which shows that $\mathrm{n}^{\prime}(t) \neq 0$ in general cases. However, the above differential equation guarantees that if $\mathrm{s}\left(t_{0}\right)+\mathrm{i}\left(t_{0}\right)+\mathrm{r}\left(t_{0}\right)=1$ for some $t_{0} \in \mathbb{R}_{\geq 0}$, then $\mathrm{s}(t)+\mathrm{i}(t)+\mathrm{r}(t)=1$ for all $t \geq t_{0}$. Accordingly, the manifold

$$
\mathcal{D}:=\{\mathrm{s}+\mathrm{i}+\mathrm{r}=1, \mathrm{~s} \geq 0, \mathrm{i} \geq 0, \mathrm{r} \geq 0\}
$$

is forward-invariant, since the flow along its boundaries is directed towards the interior-see [35] (this reduction of the state space is important, since otherwise we get an additional fixed point obtained from the first factor above, which turns out not to satisfy the conservation equation, and makes no sense from an epidemiologic point of view). Note that the conservation equation may replace either the last or the first equation in the dynamics, and allows to reduce the computation of fixed points to dimension 2.

The study of the dynamics in Proposition 1 is quite challenging, and it may sometimes be useful to also consider the two approximations introduced in the following definition.

Definition 1. Let $\Phi_{s}, \Phi_{i}, \Phi_{r} \in\{0,1\}$ and let

$$
\begin{aligned}
& \mathrm{s}^{\prime}(t)=\Lambda-\beta \mathrm{s}(t) \mathrm{i}(t)+\gamma_{r} \mathrm{r}(t)-\left(\gamma_{s}+\Lambda\right) \mathrm{s}(t)+\Phi_{s} \mathrm{~s}(t)\left(v_{i} \mathrm{i}(t)+v_{r} \mathrm{r}(t)\right), \\
& \mathrm{i}^{\prime}(t)=\mathrm{i}(t)\left[\beta \mathrm{s}(t)+\beta_{r} \mathrm{r}(t)-\left(\gamma+v_{i}+\Lambda\right)\right]+\Phi_{i} \mathrm{i}(t)\left(v_{i} \mathrm{i}(t)+v_{r} \mathrm{r}(t)\right), \\
& \mathrm{r}^{\prime}(t)=\gamma \mathrm{i}(t)+\gamma_{s} \mathrm{~s}(t)-\left(\gamma_{r}+\Lambda+v_{r}\right) \mathrm{r}(t)-\beta_{r} \mathrm{r}(t) \mathrm{i}(t)+\Phi_{r} \mathrm{r}(t)\left(v_{i} \mathrm{i}(t)+v_{r} \mathrm{r}(t)\right), \\
& \mathrm{s}(t)+\mathrm{i}(t)+\mathrm{r}(t)=1 .
\end{aligned}
$$

1. The model (11) with $\Phi_{s}=\Phi_{i}=\Phi_{r}=1$ will be called the scaled model (SM). 
2. The model (11) with $\Phi_{s}=\Phi_{i}=\Phi_{r}=0$ will be called the first approximation (FA) in the specific case of $\Lambda=\mu$. The FA is thus:

$$
\begin{aligned}
& \mathrm{s}^{\prime}(t)=\Lambda-\beta \mathrm{s}(t) \mathrm{i}(t)+\gamma_{r} \mathrm{r}(t)-\left(\gamma_{s}+\Lambda\right) \mathrm{s}(t), \\
& \mathrm{i}^{\prime}(t)=\mathrm{i}(t)\left[\beta \mathrm{s}(t)+\beta_{r} \mathrm{r}(t)-\left(\gamma+v_{i}+\Lambda\right)\right], \\
& \mathrm{r}^{\prime}(t)=\gamma \mathrm{i}(t)+\gamma_{s} \mathrm{~s}(t)-\left(\gamma_{r}+\Lambda+v_{r}\right) \mathrm{r}(t)-\beta_{r} \mathrm{r}(t) \mathrm{i}(t), \\
& \mathrm{s}(t)+\mathrm{i}(t)+\mathrm{r}(t)=1 .
\end{aligned}
$$

3. The model (11) with $\Phi_{s}=\Phi_{r}=1$ and $\Phi_{i}=0$ will be called an intermediate approximation (IA).

Remark 10. Each model, particularly $S I R / V+S$, has a $S M, F A$, and $I A$ version, which will be denoted by $S I R / V+S-S M, S I R / V+S-F A$, and $S I R / V+S-I A$.

Remark 11. The FA is not a constant population model when $v_{c}>0$, for some compartment $c$.

Remark 12. It follows from $N^{\prime}(t) / N(t)=(\Lambda-\mu)-v_{i} \mathrm{i}(t)-v_{r} \mathrm{r}(t)$ that $N^{\prime}(t)=0$ for all $t \in \mathbb{R}_{\geq 0}$ if, and only if $\mu=\Lambda$ and $v_{i}=v_{r}=0$. Thus, the popular assumption of constant population size [43] applies only to epidemics without extra deaths, which contradicts the essence of most epidemics [5]. Clearly, constant population papers have in mind some large $N$ or short-term approximation, but this is rather vague. On the other hand, the FA approximation (12) with $\mu=\Lambda$, as well as IA, may be heuristically justified as approximations obtained by ignoring certain quadratic terms in (9). This justifies studying FA without restrictive assumptions like $v_{i}=0$.

Remark 13. A considerable part of the epidemics literature has studied (1) with $N(t)=1$ (this produces an analog of (12) with $\mu \neq \Lambda$ ). The justification for studying this model is of course an assumption that $N$ is "approximately constant". The purpose of our paper is not to assume that; however, we chose to include results about them, under the name of "classic/pedagogic models" $(P M)$, to be in line with this part of the literature. As explained, we need here only results on the FA model (which approximate the object of interest to us (9)), and these may be easily recovered by replacing $\mu$ with $\Lambda$.

We conclude this section by illustrating in Figure 1 a comparison between the trajectories of the first approximation, of the intermediate approximation, and of the scaled model. Note that the approximate dynamics are an accurate approximation of the SM at the beginning of the epidemic (i.e., when $\mathrm{i}(t) \approx 0$ ). This period starts with the lower part in Figure 1, and continues until the processes start turning towards their distinct endemic points-see [26] for a rigorous slow-fast analysis of similar models. (On the other hand, in real-life controlled epidemics, for example via ICU constraints (see [44,45], etc), one has, at least for the French situation with 400 positives out of 100,000 individuals (which is still 4 to 10 times the admissible figures for Japan or other countries), that $i_{\max } \approx 0.004$. Thus, one may argue that if state upper constraints are imposed, $i \approx 0$ for all time, not just the beginning.

The Disease-Free System and Its Equilibria

It is convenient to eliminate $r$ from $s+i+r=1$, and work with the following two-dimensional scaled dynamic

$$
\left\{\begin{array}{l}
\mathrm{s}^{\prime}(t)=\Lambda-\mathrm{s}(t) \mathrm{i}(t)\left(\beta-v_{i}\right)-\left(\gamma_{s}+\Lambda\right) \mathrm{s}(t)+\left(\gamma_{r}+v_{r} \mathrm{~s}(t)\right)(1-\mathrm{s}(t)-\mathrm{i}(t)) \\
\mathrm{i}^{\prime}(t)=\mathrm{i}(t)\left[\mathrm{s}(t) \beta+v_{i} \mathrm{i}(t)+\left(v_{r}+\beta_{r}\right)(1-\mathrm{s}(t)-\mathrm{i}(t))\right]-\mathrm{i}(t)\left(\Lambda+\gamma+v_{i}\right),
\end{array}\right.
$$

defined on the positively invariant region [35]

$$
\mathcal{D}=\{(s, i), s \geq 0, i \geq 0, s+i \leq 1\} .
$$


See Figure 2.

The fixed points are the solutions of

$$
\left\{\begin{array}{c}
\mathrm{s}\left[v_{r} \mathrm{~s}+\mathrm{i}\left(\beta-v_{i}+v_{r}\right)+\Lambda+\gamma_{s}+\gamma_{r}-v_{r}\right]+\mathrm{i} \gamma_{r}-\left(\Lambda+\gamma_{r}\right)=0 \\
\mathrm{i}\left[\mathrm{s}\left(\beta-v_{r}-\beta_{r}\right)+\left(v_{i}-v_{r}-\beta_{r}\right) \mathrm{i}+v_{r}+\beta_{r}-\left(\Lambda+\gamma+v_{i}\right)\right]=0 .
\end{array}\right.
$$

The disease-free system ( with $i=0, r=1-s$ ) reduces to

$$
\begin{aligned}
\mathrm{s}^{\prime}(t) & =\Lambda-\left(\gamma_{s}+\Lambda\right) \mathrm{s}(t)+\left(\gamma_{r}+v_{r} \mathrm{~s}(t)\right)(1-\mathrm{s}(t)) \\
& =\Lambda+\gamma_{r}-\left(\gamma_{s}+\gamma_{r}+\Lambda-v_{r}\right) \mathrm{s}(t)-v_{r} \mathrm{~s}(t)^{2},
\end{aligned}
$$

and its fixed points are such that $\mathrm{s}$ satisfies the equation

$$
\left\{\begin{array}{ll}
\Lambda+\gamma_{r}-\mathrm{s}\left[v_{r} \mathrm{~s}+\Lambda+\gamma_{r}+\gamma_{s}-v_{r}\right]=0 & v_{r}>0 \\
\mathrm{~s}\left[\Lambda+\gamma_{r}+\gamma_{s}\right]-\left(\Lambda+\gamma_{r}\right)=0 & v_{r}=0
\end{array} .\right.
$$

One root

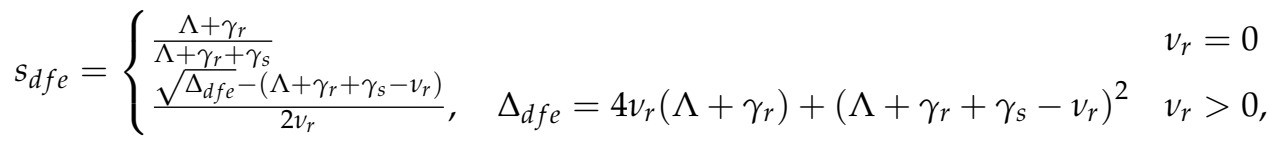

is always in $[0,1]$ and will be denoted by $s_{d f e}$.

Remark 14. $s_{d f e}$ is continuous in $v_{r}$, since for small $v_{r}$,

$$
s_{d f e} \approx \frac{\Lambda+\gamma_{r}+\gamma_{s}-v_{r}+\frac{2 v_{r}\left(\Lambda+\gamma_{r}\right)}{\Lambda+\gamma_{r}+\gamma_{s}-v_{r}}-\left(\Lambda+\gamma_{r}+\gamma_{s}-v_{r}\right)}{2 v_{r}} \rightarrow \frac{\Lambda+\gamma_{r}}{\Lambda+\gamma_{r}+\gamma_{s}} .
$$

Remark 15. The other root in the quadratic case $v_{r}>0$

$$
\frac{v_{r}-\left(\Lambda+\gamma_{r}+\gamma_{s}\right)-\sqrt{4 v_{r}\left(\Lambda+\gamma_{r}\right)+\left(\Lambda+\gamma_{r}+\gamma_{s}-v_{r}\right)^{2}}}{2 v_{r}}
$$

is strictly negative, unless

$$
\left\{\begin{array} { l } 
{ \Lambda + \gamma _ { r } = 0 } \\
{ v _ { r } \geq \gamma _ { s } + \Lambda + \gamma _ { r } }
\end{array} \Leftrightarrow \left\{\begin{array}{l}
\Lambda=\gamma_{r}=0 \\
v_{r} \geq \gamma_{s}
\end{array},\right.\right.
$$

in which case it yields a second DFE point with $\mathrm{s}=0=\mathrm{i}$.

Assumption 1. From now on, we will exclude the particular boundary case (18), which may be resolved by elementary explicit eigenvalue computations - see [35] (note, however, that while not necessarily interesting from an epidemics point of view, this case is remarkable mathematically. (More precisely, the extra DFE point $(0,0)$ may be either source- or saddle-point, and there are two endemic points, which may be either a sink and a saddle, or two sinks [35]; finally, for the general $S I R / V+S$ model, both cases may be achieved as small perturbations of the particular case (18))).

\section{DFE Stability}

Recall (cf. Assumption 1) that we exclude the case $\Lambda=\gamma_{r}=0, v_{r} \geq \gamma_{s}$ (18), so that the DFE defined in (16) is unique.

\subsection{Local DFE Stability}

The Jacobian of (13) (with r eliminated) is 


$$
J=\left(\begin{array}{cc}
-i\left(\beta-v_{i}+v_{r}\right)-\gamma_{s}-\Lambda-\gamma_{r}+v_{r}-2 s v_{r} & -s\left(\beta-v_{i}+v_{r}\right)-\gamma_{r} \\
i\left(\beta-\beta_{r}-v_{r}\right) & s\left(\beta-\beta_{r}-v_{r}\right)-\Lambda-\gamma+(2 i-1)\left(v_{i}-v_{r}-\beta_{r}\right)
\end{array}\right)
$$

Plugging in $i=0$ yields

$$
J_{d f e}=\left(\begin{array}{cc}
-2 s v_{r}+v_{r}-\Lambda-\gamma_{r}-\gamma_{s} & -s\left(\beta-v_{i}+v_{r}\right)-\gamma_{r} \\
0 & s\left(\beta-v_{r}-\beta_{r}\right)-\gamma-\Lambda-v_{i}+v_{r}+\beta_{r}
\end{array}\right),
$$

with eigenvalues

$$
\left\{\begin{array}{l}
\lambda_{P}=s_{d f e} \beta+\left(v_{r}+\beta_{r}\right) r_{d f e}-\gamma-v_{i}-\Lambda \\
\lambda_{-}=v_{r}\left(1-2 s_{d f e}\right)-\Lambda-\gamma_{r}-\gamma_{s}
\end{array} .\right.
$$

Since the DFE is unique, we may apply the next-generation matrix method [46], the first step of which consists in checking the local asymptotic stability of $s_{d f e}$ for the disease-free Equation (15). This amounts to proving that

$$
\lambda_{-}=v_{r}-\left(\Lambda+\gamma_{r}+\gamma_{s}\right)-2 v_{r} s_{d f e}<0 \text {. }
$$

However, this is automatic both when $v_{r}=0$, and when $v_{r}>0$, since by (16) $\lambda_{-}=$ $-\sqrt{\Delta_{d f e}}<0$.

With local stability in the disease-free space established, one might use the nextgeneration matrix approach. However, the direct approach is quicker here.

Proposition 2. The DFE is locally stable if the Perron-Frobenius eigenvalue of the nextgeneration matrix satisfies

$$
\mathcal{R}_{0}:=\lambda_{P}\left(F V^{-1}\right)=\frac{\beta s_{d f e}+\left(v_{r}+\beta_{r}\right) r_{d f e}}{\gamma+v_{i}+\Lambda}<1
$$

and is unstable if $\mathcal{R}_{0}>1$.

Proof. Since $\lambda_{-}<0$ by (21), the DFE is locally stable if

$$
\lambda_{P}<0 \Leftrightarrow \frac{s_{d f e} \beta+\left(v_{r}+\beta_{r}\right) r_{d f e}}{\Lambda+\gamma+v_{i}}<1 .
$$

Remark 16. The equality $\mathcal{R}_{0}=1$ is linear in $\gamma_{s}$ and yields

$$
\gamma_{s}=\frac{\left(\Lambda+v_{i}+\gamma-\beta\right)\left(v_{r}\left(\gamma+v_{i}-\gamma_{r}-v_{r}\right)-\beta_{r}\left(\Lambda+\gamma_{r}+v_{r}\right)+\beta\left(\Lambda+\gamma_{r}\right)\right)}{\left(\beta-\beta_{r}-v_{r}\right)\left(-\gamma-v_{i}-\Lambda+\beta_{r}+v_{r}\right)}:=\gamma_{s}^{*},
$$

provided that the denominator does not blow up. The parameter $\gamma_{s}^{*}$ is called "critical vaccination".

The daunting Formula (24) simplifies and turns out to provide crucial help for stability analysis in the following particular cases (this should be true in general, but we have not been able to work analytically with this daunting formula).

$$
\left\{\begin{array}{l}
v_{r}=0 \quad \Longrightarrow \frac{\gamma_{s}^{*}}{\Lambda+\gamma_{r}}=\frac{\beta-\left(\Lambda+\gamma+v_{i}\right)}{\Lambda+\gamma+v_{i}-\beta_{r}} \\
\beta_{r}=0 \quad \Longrightarrow \frac{\gamma_{s}^{*}}{\Lambda+\gamma_{r}}=\mathcal{R}-1, \mathcal{R}=\frac{\beta}{\Lambda+\gamma+v_{i}}
\end{array}\right.
$$

The first formula is positive if either $\beta_{r} \leq \Lambda+\gamma+v_{i} \leq \beta$, or $\beta \leq \Lambda+\gamma+v_{i} \leq \beta_{r}$, and a geometric consequence of this is provided in Section 5. 


\subsection{Global Stability of the Disease-Free Equilibrium}

Proposition 3. If $\mathcal{R}_{0}<1$ and DFE is the unique equilibrium point, then it is globally asymptotically stable in the invariant set $\mathcal{D}$.

Proof. Each solution starting in $\mathcal{D}$ is obviously bounded, so its $\omega$-limit set is not empty. The Poincare-Bendixon Theorem implies that this is the unique equilibrium point DFE, since otherwise it would be a closed orbit (see [47]) and one may check similarly as in [Theorem 3.1] of [32] that no periodic orbits exist.

Since the explicit conditions for the uniqueness of the equilibrium point are quite complicated, we prefer to provide them only in a particular case, in Section 5.

For the general case, we may find a simple criteria if we weaken the concept of global stability of the disease-free Equilibrium as follows:

Proposition 4. If

$$
\max \left[\beta_{r}+v_{r}, \beta, \Lambda+\gamma+v_{i}\right]=\Lambda+\gamma+v_{i},
$$

then the DFE is "strongly globally stable", in the sense that the function $L(\mathrm{~s}, \mathrm{i})=\mathrm{i}$ is Lyapunov over the invariant region $\mathcal{D}:=\left\{(\mathrm{s}, \mathrm{i}) \in \mathbb{R}_{+}^{2}: 0 \leq \mathrm{s}+\mathrm{i} \leq 1\right\}$.

Remark 17. In lay-terms, we may say that "the epidemics never picks up" in this case.

Proof. The non-negative function $L(\mathrm{~s}, \mathrm{i})=\mathrm{i}$, with $L(1,0)=0$, is Lyapunov (i.e., the epidemics may never increase) if

$$
L^{\prime}(\mathrm{s}, \mathrm{i})=\mathrm{i}^{\prime} \leq 0 \Leftrightarrow s\left(\beta-\beta_{r}-v_{r}\right)+\left(v_{i}-v_{r}-\beta_{r}\right) i+\beta_{r}+v_{r}-\Lambda-\gamma-v_{i} \leq 0 .
$$

The maximimization of $i^{\prime}$ thus reduces to a simple linear programming problem. Thus, there must exist an extremal point of $\mathcal{D}$ where the maximum is attained, and

$$
\begin{aligned}
& \max _{(s, i) \in \mathcal{D}} s\left(\beta-\beta_{r}-v_{r}\right)+\left(v_{i}-v_{r}-\beta_{r}\right) i+\beta_{r}+v_{r}-\Lambda-\gamma-v_{i}= \\
& \max \left[\beta_{r}+v_{r}-\Lambda-\gamma-v_{i}, \beta-\Lambda-\gamma-v_{i},-\Lambda-\gamma\right] \leq 0,
\end{aligned}
$$

provided that

$$
\beta_{r}+v_{r} \leq \Lambda+\gamma+v_{i}, \mathcal{R}_{0}<1 \Leftrightarrow \max \left[\beta_{r}+v_{r}, \beta, \Lambda+\gamma+v_{i}\right]=\Lambda+\gamma+v_{i}
$$

\section{The Endemic Equilibria}

The endemic equilibrium set may be obtained algebraically by solving one of the variables from $\mathrm{i}^{\prime} / \mathrm{i}=0$ in (14), and plugging it into the other equation. Eliminating $\mathrm{s}$ using

$$
s=1-i+\frac{i\left(v_{i}-\beta\right)+\beta-\left(\Lambda+\gamma+v_{i}\right)}{-\beta+\beta_{r}+v_{r}}, \beta \neq \beta_{r}+v_{r}
$$

yields a quadratic equation $A i^{2}+B i+C=0$, where $A=\left(\beta-\beta_{r}\right)\left(\beta-v_{i}\right)\left(\beta_{r}+v_{r}-v_{i}\right)$, and the other coefficients are very complicated.

Thus, in the complex plane, $i_{1,2}^{(E E)}=\frac{-B \pm \sqrt{\Delta}}{2 A}$. Since analytically locating the endemic points requires considerable effort, we will restrict starting with the next section to the case $v_{r}=0$.

Remark 18. However, we note already that: 
1. The equation is quadratic if $\beta \neq \beta_{r}, \beta \neq v_{i}$ and $\beta_{r}+v_{r} \neq v_{i}$. We will at first exclude these particular cases, but note that they suggest that different regimes occur when the respective thresholds are crossed - this will be further explained below.

2. Locating the endemic points may be achieved geometrically by studying the intersection of the vertical and horizontal isoclines:

(a) the $i^{\prime}=0$ isocline is given by $i=0$ and by the herd immunity line

$$
s\left(\beta-\beta_{r}-v_{r}\right)+i\left(v_{i}-\beta_{r}-v_{r}\right)=\Lambda+\gamma+v_{i}-\beta_{r}-v_{r}
$$

with slope $\frac{\beta-\beta_{r}-v_{r}}{\beta_{r}+v_{r}-v_{i}}$.

(b) The $s^{\prime}=0$ isocline is

$$
\frac{v_{r}}{\Lambda+\gamma_{r}+\gamma_{s}} s^{2}+i s \frac{\beta-v_{i}+v_{r}}{\Lambda+\gamma_{r}+\gamma_{s}}+s+i \frac{\gamma_{r}}{\Lambda+\gamma_{r}+\gamma_{s}}-\frac{\Lambda+\gamma_{r}}{\Lambda+\gamma_{r}+\gamma_{s}}=0
$$

which is a hyperbola.

This passes by definition through the DFE, and therefore necessarily intersects the domain when

$$
s_{d f e}<1 \Leftrightarrow \gamma_{s}>0
$$

\section{The Case $v_{r}=0, \gamma_{s}>0$}

Getting sharper stability results beyond the weak $\mathcal{R}_{0}$ alternative requires locating the endemic points, and this seems quite difficult in general. Therefore, we will restrict from now on to the case $v_{r}=0$, which avoids the necessity of handling the complications arising from the square root formula of $s_{d f e}$. The case $\gamma_{s}=0$, essentially covered in [Section 4] of [32] , requires special treatment-see Section 6.

The quadratic equation $A i^{2}+B i+C=0$ has coefficients

$$
\left\{\begin{array}{l}
C=-\left(\gamma+v_{i}+\Lambda\right)\left(\Lambda+\gamma_{r}+\gamma_{s}\right)+\beta\left(\Lambda+\gamma_{r}\right)+\beta_{r} \gamma_{s} \\
B=\beta\left(-\gamma-v_{i}-\Lambda+\beta_{r}-\gamma_{r}\right)-\beta_{r}\left(v_{i}+\Lambda+\gamma_{s}\right)+v_{i}\left(\gamma+v_{i}+2 \Lambda+\gamma_{r}+\gamma_{s}\right) \\
A=\left(\beta-v_{i}\right)\left(v_{i}-\beta_{r}\right) .
\end{array}\right.
$$

The endemic points are still complicated:

$$
E E_{1,2}=\left\{\begin{array}{l}
s=\frac{\beta\left(\gamma+v_{i}+\Lambda-\beta_{r}-\gamma_{r}\right)-v_{i}\left(\gamma+v_{i}-\beta_{r}-\gamma_{r}-\gamma_{s}\right)-\beta_{r}\left(\Lambda+\gamma_{s}\right) \pm \sqrt{\Delta}}{2\left(\beta-\beta_{r}\right)\left(\beta-v_{i}\right)} \\
i=\frac{\beta \gamma+\beta \Lambda+\beta v_{i}-\gamma v_{i}-2 \Lambda v_{i}-v_{i}^{2}+v_{i} \beta_{r}+\Lambda \beta_{r}-\beta \beta_{r} \mp \sqrt{\Delta}}{2\left(v_{i}-\beta\right)\left(\beta_{r}-v_{i}\right)}
\end{array},\right.
$$

where

$$
\begin{aligned}
& \Delta=4\left(\beta-\beta_{r}\right)\left(\beta-v_{i}\right)\left(\Lambda\left(-v_{i}+\beta_{r}+v_{r}\right)+(\gamma+\mu) \gamma_{r}\right) \\
& +\left(\beta\left(-\gamma-v_{i}-\mu+\beta_{r}+\gamma_{r}+v_{r}\right)+\beta_{r}\left(-v_{i}+\mu+\gamma_{s}\right)+v_{i}\left(\gamma+v_{i}-\gamma_{r}-\gamma_{s}\right)-v_{r}\left(\gamma+v_{i}-\gamma_{s}\right)\right)^{2}
\end{aligned}
$$

By solving $C=0$, we may compute, however, an important critical value for $\beta$,

$$
\beta_{*}=\frac{\left(\gamma+v_{i}+\Lambda\right)\left(\Lambda+\gamma_{r}+\gamma_{s}\right)-\beta_{r} \gamma_{s}}{\Lambda+\gamma_{r}}
$$

above which one (the higher) of the endemic points crosses above the $i=0$ axis, therefore entering $\mathcal{D}$ (equivalently, $\mathcal{R}_{0}$ becomes larger than 1 ).

Locating the endemic points may be attempted algebraically-see Lemma A2 in the Appendix, which seemed quite difficult.

We therefore resorted to a geometric study of the isoclines in Theorem 1, attempting to geometrically explain all the possible cases. For example, the case $\mathcal{R}_{0}>1$ turned out to 
be equivalent to the unicity of the endemic point, and to the fact that the immunity line intersects with the domain line on both sides of the $s^{\prime}=0$ isocline.

Remark 19. Since the number of crossing points must be odd, on one hand, and less than two on the other, identifying such a crossing is equivalent to the uniqueness of the endemic point.

We turn now to listing some elementary geometric facts, particularly the coordinates of various intersection points.

1. When $v_{i} \neq \beta$, the $s^{\prime}=0$ isocline becomes the hyperbola

$$
\begin{aligned}
& s_{d f e}=i s \frac{\beta-v_{i}}{\Lambda+\gamma_{r}+\gamma_{s}}+\frac{\gamma_{r}}{\Lambda+\gamma_{r}+\gamma_{s}} i+s \\
& =i \frac{\beta-v_{i}}{\Lambda+\gamma_{r}+\gamma_{s}}\left(s+\frac{\gamma_{r}}{\beta-v_{i}}\right)+s+\frac{\gamma_{r}}{\beta-v_{i}}-\frac{\gamma_{r}}{\beta-v_{i}} \\
& \Leftrightarrow\left(i \frac{\beta-v_{i}}{\Lambda+\gamma_{r}+\gamma_{s}}+1\right)\left(s+\frac{\gamma_{r}}{\beta-v_{i}}\right)=s_{d f e}+\frac{\gamma_{r}}{\beta-v_{i}},
\end{aligned}
$$

with asymptotes $s=\frac{\gamma_{r}}{v_{i}-\beta}, i=\frac{\Lambda+\gamma_{r}+\gamma_{s}}{v_{i}-\beta}$. Note that the center is in the first quadrant when $\beta<v_{i}$ and in the third quadrant otherwise, and that the intersection $E$ with the line $s=0$ has $i_{E}=1+\frac{\Lambda}{\gamma_{r}}$, outside $\mathcal{D}$. The convexity of the branch which intersects $\mathcal{D}$ is also important. From

$$
i^{\prime \prime}\left(s_{d f e}\right)=2 \frac{s_{d f e}+k}{(s+k)^{3}} \frac{\gamma_{r}+\Lambda+\gamma_{s}}{\beta-v_{i}}, k=\frac{\gamma_{r}}{\beta-v_{i}},
$$

we find that our branch is convex when $\beta>v_{i}$, and concave otherwise. The equality case $v_{i}=\beta$ is analyzed in the following remark.

Remark 20. When $v_{i}=\beta$, the $s^{\prime}=0$ hyperbola degenerates into a line $s\left(\Lambda+\gamma_{r}\right)+$ $i \gamma_{r}-\gamma_{r}-\Lambda=0$. The intersection of the two lines gives a unique endemic point $E E=$ $\left(\frac{(\gamma+\Lambda) \gamma_{r}}{\Lambda\left(\beta_{r}-v_{i}\right)}+1, \frac{(\gamma+\Lambda)\left(\Lambda+\gamma_{r}\right)}{\Lambda\left(v_{i}-\beta_{r}\right)}\right)$ which never belongs to the feasible region. Indeed, $i_{e e}>0$ if, and only if $v_{i}>\beta_{r}$, and this further implies

$$
s_{e e}+i_{e e}=1+\frac{(\gamma+\Lambda) \Lambda}{\Lambda\left(v_{i}-\beta_{r}\right)}>1 \Longrightarrow E E \notin \mathcal{D} .
$$

2. A crucial role in the analysis is played by the point where the immunity line intersects $i=0$, given by $B\left(\frac{\Lambda+v_{i}+\gamma-\beta_{r}}{\beta-\beta_{r}}, 0\right), \beta_{r} \neq \beta$ (when $\beta_{r}=\beta, B$ goes to $\infty$ ). This point satisfies:

$$
\begin{cases}s_{B}<0 & \text { if } \Lambda+v_{i}+\gamma<\beta_{r}<\beta, \text { or } \beta<\beta_{r}<\Lambda+v_{i}+\gamma \\ s_{B} \in[0,1] & \text { if } \beta \leq \Lambda+v_{i}+\gamma<\beta_{r}, \text { or } \beta_{r}<\Lambda+v_{i}+\gamma<\beta . \\ s_{B}>1 & \text { if } \beta_{r}<\beta<\Lambda+v_{i}+\gamma \text { or } \Lambda+v_{i}+\gamma<\beta<\beta_{r}\end{cases}
$$

The six cases listed above are the basis of our geometric analysis provided in Theorem 1 .

Remark 21. $s_{B}$ coincides with $s_{d f e}$ if $\gamma_{s}=\gamma_{s}^{*}$, which fits with the fact that $\gamma_{s}^{*} \in(0,1)$ if one of these two cases occurs-recall Remark 16.

3. Another important point is the point $A$ where the immunity line (27) intersects $i=1-s$, with coordinates

$$
\left(s_{A}, i_{A}\right)=\left(\frac{\gamma+\Lambda}{\beta-v_{i}}, \frac{\beta-\left(\gamma+v_{i}+\Lambda\right)}{\beta-v_{i}}\right) .
$$


It is easy to show that $A \in \mathcal{D}$ if $\beta>\Lambda+v_{i}+\gamma$, that it then moves to the fourth quadrant when $\Lambda+v_{i}+\gamma \geq \beta>v_{i}$, and that it jumps from the fourth to the second quadrant when $\beta$ decreases below the threshold $v_{i}$.

4. The point where the immunity line intersects $s=0$ is $C\left(0,1+\frac{\gamma+\Lambda}{v_{i}-\beta_{r}}\right)$. This is negative when $v_{i}<\beta_{r}<\Lambda+\gamma+v_{i}$, in the domain when $\beta_{r} \geq \Lambda+\gamma+v_{i}$, and bigger than 1 when $\beta_{r} \leq v_{i}$. More precisely,

$$
C=\frac{\Lambda+\gamma+v_{i}-\beta_{r}}{v_{i}-\beta_{r}} \text { satisfies }\left\{\begin{array}{ll}
i_{C} \leq 0 & \text { if } v_{i}<\beta_{r}<\Lambda+v_{i}+\gamma \\
i_{C} \in[0,1] & \text { if } \beta_{r} \geq \Lambda+v_{i}+\gamma \\
i_{C}>1 & \text { if } \beta_{r}<v_{i}
\end{array} .\right.
$$

5. The unique point $D$ where the hyperbola intersects $\mathrm{s}+\mathrm{i}=1$ within the domain has coordinates

$$
\left\{\begin{array}{l}
s_{D}=\frac{1}{2}+\frac{\Lambda+\gamma_{s}-\sqrt{4 \Lambda\left(v_{i}-\beta\right)+\left(\beta-v_{i}+\Lambda+\gamma_{s}\right)^{2}}}{2\left(\beta-v_{i}\right)} \\
i_{D}=\frac{1}{2}+\frac{-\Lambda-\gamma_{s}+\sqrt{4 \Lambda\left(v_{i}-\beta\right)+\left(\beta-v_{i}+\Lambda+\gamma_{s}\right)^{2}}}{2\left(\beta-v_{i}\right)}
\end{array} .\right.
$$

Remark 22. When $\beta=\beta_{r}$, the slope $\frac{\beta-\beta_{r}}{\beta_{r}-v_{i}}$ of the immunity line becomes 0 and $i_{C}=i_{A}=$ $\frac{\beta-\left(\gamma+v_{i}+\Lambda\right)}{\beta-v_{i}}=\frac{1}{\gamma+v_{i}+\Lambda} \frac{\mathcal{R}_{0}-1}{\beta-v_{i}}$. The dynamical system admits a unique endemic point and a unique EE given by

$$
E E=\left(\frac{\Lambda\left(\beta-v_{i}+\gamma_{r}\right)+\gamma \gamma_{r}}{\left(\beta-v_{i}\right)\left(\beta-\gamma-v_{i}+\gamma_{r}+\gamma_{s}\right)}, \frac{\beta-\Lambda+\gamma+v_{i}}{\beta-v_{i}}\right),
$$

which may be checked to belong to the feasible region if

$$
\mathcal{R}=\frac{\beta}{\Lambda+\gamma+v_{i}}>1
$$

Indeed, $i_{e e}>0$ requires either $\beta<v_{i}$, which leads subsequently to a contradiction, or $\beta>$ $\Lambda+\gamma+v_{i}$, which may be shown to imply $s>0, s+i<1$. The stability analysis of this case is elementary and left to the reader.

The Formula (32) and the subsequent Remark 21 suggest splitting the analysis according to the order of the three quantities $\beta, \Lambda+\gamma+v_{i}, \beta_{r}$ and on the orders of $\gamma_{s}, \gamma_{s}^{*}=$ $\left(\Lambda+\gamma_{r}\right) \frac{\beta-\left(\Lambda+\gamma+v_{i}\right)}{\Lambda+\gamma+v_{i}-\beta_{r}}$ and of $\left(v_{i}, \beta\right)$. We end up with 10 cases, nine of which are fully resolved in Theorem 1, and one of which is left partly open. Note that, as proved in the Appendix, these 10 cases form a disjoint decomposition of the parameter space if only strict inequalities are allowed.

Before stating Theorem 1, we provide graphical illustrations of the 10 cases.

Theorem 1. Suppose $v_{r}=0$, and that neither two of the three parameters $\beta, \beta_{r}, v_{i}$ coincide. Then, one of the following cases must arise:

1. $\mathcal{R}_{0}>1 \Leftrightarrow$ precisely, one endemic point lies in $\mathcal{D}$, which may occur in one of the following four ways:

(a) $\quad A C \in \mathcal{D}$ crosses the hyperbola with positive slope $\Lambda+\gamma+v_{i} \leq \beta_{r}<\beta-$ see Figure 3a;

(b) $\quad A C \in \mathcal{D}$ crosses the hyperbola with negative slope $\Lambda+\gamma+v_{i} \leq \beta<\beta_{r}$-see Figure $3 b$;

(c) $\quad \beta_{r} \leq \Lambda+\gamma+v_{i} \leq \beta, \gamma_{s}<\gamma_{s}^{*} \Leftrightarrow B A \in \mathcal{D}$ crosses the hyperbola-Figure $3 c$;

(d) $\beta \leq \Lambda+\gamma+v_{i} \leq \beta_{r}, \gamma_{s} \geq \gamma_{s}^{*} \Leftrightarrow B C \in \mathcal{D}$ crosses the hyperbola-Figure $3 d$.

In all these cases, the endemic point is a sink and DFE is a saddle point. 
2. $\mathcal{R}_{0} \leq 1$ is the union of the following six cases (similar to the above, but taking also into account the convexity of the hyperbola branch, in some cases):

(a)

$$
\beta_{r}<\Lambda+\gamma+v_{i}<\beta, v_{i}<\beta,
$$

when both the points $A, B$ lie to the right of a convex hyperbola branch, and the isoclines do not intersect in $\mathcal{D}$-see Figure $4 a$.

(b)

$$
\beta<v_{i}<\Lambda+\gamma+v_{i}<\beta_{r}
$$

when both the points $C, B$ lie to the left of a concave hyperbola branch, and the isoclines do not intersect in $\mathcal{D}$-see Figure $4 b$.

(c) When both the points C, B lie to the left of a convex hyperbola branch, we have two subcases:

i. When

$$
v_{i}<\beta<\Lambda+\gamma+v_{i}<\beta_{r}, \gamma_{s}<\gamma_{s}^{*}, \Delta<0,
$$

the isoclines intersect $\mathcal{D}$, but do not intersect each other-see Figure $4 c$.

ii. The isoclines intersect in $\mathcal{D}$, yielding two endemic points-see Figure $4 d$. Necessary conditions for this are

$$
v_{i}<\beta<\Lambda+\gamma+v_{i}<\beta_{r}, \gamma_{s}<\gamma_{s}^{*}, \Delta \geq 0,
$$

and we conjecture that the necessary and sufficient conditions are obtained by adding $\beta_{r} \geq \beta_{r}^{(+)}$, where $\beta_{r}^{(+)}$is defined in (36).

In this case, the DFE is one of two sink points, whose attraction domains are separated by the separatrices of the third fixed saddle point.

(d) The $i^{\prime} / i=0$ isocline does not intersect the interior of $\mathcal{D}$ in the following two cases:

i. $\quad \beta \leq \beta_{r} \leq \Lambda+\gamma+v_{i}$, with hyperbole concave-see Figure 5a-or convex, according to whether $\beta>v_{i}$ or not;

ii. $\beta_{r}<\beta \leq \Lambda+\gamma+v_{i}$, with hyperbole convex-see Figure $5 b$-or convave, according to whether $\beta<v_{i}$ or not;

3. In all the cases when the DFE is the unique fixed point within the domain, it is a globally stable sink.

\section{Proof.}

1. As noted already, $\beta>\Lambda+\gamma+v_{i}$ is equivalent to fact that the point $A=\left(\frac{\gamma+\Lambda}{\beta-v_{i}}, \frac{\beta-v_{i}-\gamma-\Lambda}{\beta-v_{i}}\right)$ lies in $\mathcal{D}$, which applies in the cases $1(\mathrm{a}-\mathrm{c})$. Furthermore, we may check that in this case, the point $A$ is always to the right of (above) the hyperbola, that is,

$$
s_{D}=\frac{1}{2}+\frac{\Lambda+\gamma_{s}-\sqrt{4 \Lambda\left(v_{i}-\beta\right)+\left(\beta-v_{i}+\Lambda+\gamma_{s}\right)^{2}}}{2\left(\beta-v_{i}\right)}<\frac{\gamma+\Lambda}{\beta-v_{i}}=s_{A} .
$$

To conclude the existence of a unique endemic point, it is thus enough to find in these three cases a point of the immunity line below the hyperbola. Referring to Figure 3, we see that the following cases may arise:

(a) $\quad A C \in \mathcal{D}$ crosses the hyperbola, and $s_{B}<0$. We must then be in the case $\Lambda+\gamma+v_{i}<\beta_{r}<\beta$, which implies $\beta_{r} \geq \Lambda+v_{i}+\gamma$, and so $C \in \mathcal{D}$ is below the hyperbola-see Figure 3a.

(b) $\quad A C \in \mathcal{D}$ crosses the hyperbola, and $s_{B} \geq 1$. We must then be in the case $\Lambda+\gamma+v_{i}<\beta<\beta_{r}$, which implies again $\beta_{r} \geq \Lambda+v_{i}+\gamma$, and so $C \in \mathcal{D}$ is again below the hyperbola-see Figure $3 \mathrm{~b}$.

(c) $\quad B A \in \mathcal{D}$ crosses the hyperbola when $\mathcal{R}_{0}>1, \beta>\Lambda+\gamma+v_{i}>\beta_{r}, s_{B} \leq$ $s_{d f e} \Leftrightarrow \gamma_{s} \leq \gamma_{s}^{*}$, since $B$ is below the hyperbola-see Figure 3c. 
(d) $\quad B C \in \mathcal{D}$ crosses the hyperbola (and $A \notin \mathcal{D}$ ) when $\mathcal{R}_{0}>1, \beta_{r}>\Lambda+\gamma+v_{i}>$ $\beta, s_{B}>s_{d f e} \Leftrightarrow \gamma_{s}>\gamma_{s}^{*}$, since $B$ is below the hyperbola-see Figure $3 \mathrm{~d}$.

2. We turn now to the case $\mathcal{R}_{0} \leq 1$, when the point $A$ lies in the fourth quadrant if $v_{i}<\beta$, and in the second quadrant otherwise.

(a) In this case $s_{B}>s_{d f e}, s_{A}>s_{D}$. Since the immunity line is to the right of a convex hyperbola branch, it is clear geometrically that they cannot intersect within the domain-see Figure 4 a.

(b) Similarly, the end points in $\mathcal{D}$ of the immunity line are to the left of a concave hyperbola branch, and so they cannot intersect within the domain-see Figure $4 \mathrm{~b}$.

(c) For two endemic points to exist, it is necessary that $s_{B}=\frac{\Lambda+v_{i}+\gamma-\beta_{r}}{\beta-\beta_{r}} \in\left(0, s_{d f e}\right)$, which requires that

$$
\beta \leq \Lambda+\gamma+v_{i} \leq \beta_{r}
$$

and this implies that the other intercept $C=\left(0, \frac{\Lambda+\gamma+v_{i}-\beta_{r}}{v_{i}-\beta_{r}}\right)$ is also in $\mathcal{D}$.

The $i^{\prime} / i=0$ isocline intersects then $\mathcal{D}$, and may also intersect the hyperbola, when the discriminant

$$
\begin{aligned}
& \Delta=\left(v_{i}\left(v_{i}-\beta_{r}+\gamma-\gamma_{r}-\gamma_{s}\right)+\beta_{r}\left(\gamma_{s}+\Lambda\right)+\beta\left(\beta_{r}-\gamma+\gamma_{r}-\Lambda-v_{i}\right)\right)^{2} \\
& +4\left(\beta-\beta_{r}\right)\left(\beta-v_{i}\right)\left(\gamma \gamma_{r}+\Lambda\left(\beta_{r}+\gamma_{r}-v_{i}\right)\right)
\end{aligned}
$$

satisfies $\Delta \geq 0$.

The inequality $\Delta>0$ is quadratic in $\beta_{r}$ and may be rewritten as

$$
\begin{aligned}
& \beta_{r}+\Lambda+\gamma_{r}-v_{i}+\frac{\Lambda\left(\Lambda+\gamma_{r}\right)}{\beta-v_{i}-\Lambda}-\frac{\gamma\left(\beta-v_{i}\right)\left(\beta-v_{i}+\Lambda+2 \gamma_{r}\right)}{\left(\beta-v_{i}-\Lambda\right)^{2}} \notin[-\mathcal{L}, \mathcal{L}] \\
& \mathcal{L}=2 \frac{\sqrt{\gamma\left(\Lambda+\gamma_{r}\right)\left(\beta-v_{i}\right)^{2}\left(\beta-v_{i}+\gamma_{r}\right)\left(\Lambda+\gamma+v_{i}-\beta\right)}}{\left(\beta-v_{i}-\Lambda+\gamma_{s}\right)^{2}+4 \Lambda \gamma_{s}}
\end{aligned}
$$

(note that $\mathcal{L}>0$ when $\mathcal{R}<1, v_{i}<\beta$ ).

We conjecture based on numerical evidence that the two endemic points belong to $\mathcal{D}$ only when $\beta_{r}$ is larger than the largest root $\beta_{r}^{(+)}$of $\Delta=0$, defined implicitly in (36). When $\gamma_{s}=0$, the largest root is reduced to

$$
\beta_{r}^{(+)}=\mathcal{L}+\frac{\gamma\left(\beta-v_{i}\right)\left(\beta-v_{i}+\Lambda+2 \gamma_{r}\right)-\left(\beta-v_{i}-\Lambda\right)\left(v_{i}^{2}-v_{i}\left(\beta+\gamma_{r}\right)+\beta\left(\Lambda+\gamma_{r}\right)\right)}{\left(-\beta+v_{i}+\Lambda\right)^{2}}
$$

where

$$
\mathcal{L}:=\frac{2 \sqrt{\gamma\left(\beta-v_{i}\right)^{2}\left(\Lambda+\gamma_{r}\right)\left(-\beta+\Lambda+\gamma+v_{i}\right)\left(\beta-v_{i}+\gamma_{r}\right)}}{\left(-\beta+v_{i}+\Lambda\right)^{2}}
$$

(d) In the last case, we must show that the $i^{\prime} / i=0$ isocline does not intersect the interior of $\mathcal{D}$. Equivalently, we must show that in each of the two subcases

$$
\left\{\begin{array}{l}
\beta<\beta_{r} \leq \Lambda+\gamma+v_{i} \\
\beta_{r} \leq \beta \leq \Lambda+\gamma+v_{i}
\end{array}\right.
$$

none of the points $A, B, C$ belong to $\mathcal{D}$. This is a tedious computation, and not reproduced here. For a quick check, we offer a Mathematica file on our website (we rely mostly on FindInstance with an empty output to show that certain cases do not exist, and on the command Reduce to decompose other cases into subcases). 


\section{The Boundary Case $\gamma_{s}=v_{r}=0$}

The case $\gamma_{s}=v_{r}=0$, generalizes on the particular case $\gamma_{s}=v_{r}=0=\gamma_{r}$, which was called the SIRI model in [Section 4] of [32] .

Note first that, in this case, $s_{d f e}=1$. It follows that cases 4, 5 (1(d) and 2(a)) may not arise, and case $6(2(\mathrm{~b}))$ becomes degenerate, since $s_{d f e}=1$. It now becomes possible that the hyperbola does not intersect the domain. This is equivalent to its slope at the DFE $i^{\prime}\left(s_{d f e}\right)=-\frac{\Lambda+\gamma_{r}}{\beta-v_{i}+\gamma_{r}}$ being either positive or less than -1 , and further equivalent to

$$
\beta \leq \Lambda+v_{i}
$$

This further implies $\mathcal{R}<1$, and, together with the absence of endemic points and of periodic solutions, leads to the fact that the DFE is the global attractor.

After having dealt with this case, which also includes the concave case $\beta>v_{i}$, one may restrict to the case when the hyperbola does intersect the domain, which is equivalent to its slope at the DFE being such that

$$
-1<i^{\prime}\left(s_{d f e}\right)<0 \Leftrightarrow \beta>\Lambda+v_{i}
$$

(note this implies that its center is in the third quadrant).

The proof becomes simpler than in the previous section. For example, the point $D$ of intersection of the hyperbola with $i=1-s$ satisfies

$$
s_{D}=\frac{\Lambda}{\beta-v_{i}} \in(0,1),
$$

and hence belongs to $\mathcal{D}$.

The roots of $\Delta=0$ now simplify to:

$$
\begin{aligned}
\beta_{r}^{( \pm)} & :=v_{i}-\Lambda-\gamma_{r}-\frac{\Lambda\left(\Lambda+\gamma_{r}\right)}{\beta-v_{i}-\Lambda}+\frac{\gamma\left(\beta-v_{i}\right)\left(\beta-v_{i}+\Lambda+2 \gamma_{r}\right)}{\left(\beta-v_{i}-\Lambda\right)^{2}} \pm \mathcal{L}, \\
\mathcal{L} & =2 \frac{\sqrt{\gamma\left(\Lambda+\gamma_{r}\right)\left(\beta-v_{i}\right)^{2}\left(\beta-v_{i}+\gamma_{r}\right)\left(\Lambda+\gamma+v_{i}-\beta\right)}}{\left(-\beta+v_{i}+\Lambda\right)^{2}}
\end{aligned}
$$

\section{The Classic/Pedagogical SIR/V+S Model}

The pedagogical model is defined as follows:

$$
\begin{aligned}
& \mathrm{s}^{\prime}(t)=\Lambda-\beta \mathrm{s}(t) \mathrm{i}(t)+\gamma_{r} \mathrm{r}(t)-\left(\gamma_{s}+\mu\right) \mathrm{s}(t), \\
& \mathrm{i}^{\prime}(t)=\mathrm{i}(t)\left[\beta \mathrm{s}(t)+\beta_{r} \mathrm{r}(t)-\left(\gamma+v_{i}+\mu\right)\right], \\
& \mathrm{r}^{\prime}(t)=\gamma \mathrm{i}(t)+\gamma_{s} \mathrm{~s}(t)-\left(\gamma_{r}+\mu+v_{r}\right) \mathrm{r}(t)-\beta_{r} \mathrm{r}(t) \mathrm{i}(t) .
\end{aligned}
$$

The following properties hold:

1. The region

$$
\mathcal{D}=\left\{(\mathrm{s}, \mathrm{i}, N) \in \mathbb{R}_{+}^{3}, \mathrm{~s}+\mathrm{i} \leq N \leq \Lambda / \mu\right\}
$$

may be shown to be positively invariant with respect to (38); therefore, this region must include an attractor set $[48,49]$.

2. The DFE equilibrium point of the pedagogic system is obtained by plugging $i_{d f e}=0$ into (38). Solving with respect to $(s, r)$ the remaining first and third equations

$$
\left\{\begin{array}{l}
0=\Lambda-\left(\gamma_{s}+\mu\right) \mathrm{s}+\gamma_{r} \mathrm{r} \\
0=\gamma_{s} \mathrm{~s}-\left(\gamma_{r}+\mu+v_{r}\right) \mathrm{r}
\end{array}\right.
$$


yields

$$
X^{(D F E)}=\left(\frac{\Lambda\left(\mu+\gamma_{r}+v_{r}\right)}{\mu \gamma_{r}+\left(\mu+v_{r}\right)\left(\mu+\gamma_{s}\right)}, 0, \frac{\Lambda \gamma_{s}}{\mu \gamma_{r}+\left(\mu+v_{r}\right)\left(\mu+\gamma_{s}\right)}\right) .
$$

In particular, the DFE for the FA model is obtained by substituting $\Lambda=\mu$ in (40):

$$
X^{(D F E)}=\left(\frac{\Lambda\left(\Lambda+\gamma_{r}+v_{r}\right)}{\Lambda \gamma_{r}+\left(\Lambda+v_{r}\right)\left(\Lambda+\gamma_{s}\right)}, 0, \frac{\Lambda \gamma_{s}}{\Lambda \gamma_{r}+\left(\Lambda+v_{r}\right)\left(\Lambda+\gamma_{s}\right)}\right) .
$$

Note the relation $v_{r} \gamma_{s}=0 \Longrightarrow i_{d f e}+r_{d f e}+s_{d f e}=1$.

3. When $\beta_{r}>0$, there may be two endemic equilibrium points (we omit their complicated expressions), but when $\beta_{r}=0$ there is a unique FA EE, obtained by plugging

$$
s=\frac{1}{\mathcal{R}}=\frac{\gamma+\Lambda+v_{i}}{\beta}
$$

into the first and third equations, and solving the linear system

$$
\left\{\begin{array}{l}
\Lambda+\gamma_{r} \mathrm{r}(t)-\beta \mathrm{i} / \mathcal{R}-\left(\gamma_{s}+\Lambda\right) / \mathcal{R}=0, \\
\gamma \mathrm{i}+\gamma_{s} / \mathcal{R}-\left(\gamma_{r}+\Lambda+v_{r}\right) \mathrm{r}=0
\end{array} .\right.
$$

This yields $X^{(E E)}=$

$$
\begin{gathered}
\left(\frac{1}{\mathcal{R}}, \frac{\gamma_{r}(\Lambda-\Lambda \mathcal{R})+\left(\Lambda+v_{r}\right)\left(\gamma_{s}+\Lambda-\Lambda \mathcal{R}\right)}{\gamma \gamma_{r} \mathcal{R}-\beta\left(\gamma_{r}+\Lambda+v_{r}\right)}, \frac{\gamma \mathcal{R}\left(\gamma_{s}+\Lambda-\Lambda \mathcal{R}\right)-\beta \gamma_{s}}{\mathcal{R}\left(\gamma \gamma_{r} \mathcal{R}-\beta\left(\gamma_{r}+\Lambda+v_{r}\right)\right)}\right) \\
=\left(\frac{1}{\mathcal{R}}, \frac{\mathcal{R} s_{d f e}-1}{z}, \frac{\gamma \mathcal{R}\left(\gamma_{s}+\Lambda-\Lambda \mathcal{R}\right)-\beta \gamma_{s}}{\mathcal{R}\left(\gamma \gamma_{r} \mathcal{R}-\beta\left(\gamma_{r}+\Lambda+v_{r}\right)\right)}\right)
\end{gathered}
$$

where $z:=\mathcal{R}\left[\frac{\gamma_{r}\left(\Lambda+v_{i}\right)+\left(\Lambda+v_{r}\right)\left(\gamma+\Lambda+v_{i}\right)}{\Lambda \gamma_{r}+\left(\Lambda+v_{r}\right)\left(\Lambda+\gamma_{s}\right)}\right]$.

Note the intriguing simplification of $i^{(E E)}$, which shows that

$$
i^{(E E)} \geq 0 \Leftrightarrow 1 \leq \mathcal{R} s_{d f e},
$$

and it may be shown that the endemic point belongs to the domain if $1 \leq \mathcal{R} s_{d f e}$. This gives a pre-warning on the role of the parameter $\mathcal{R} s_{d f e}:=\mathcal{R}$ in the stability of the DFE.

Example 1. In the particular case $v_{r}=\gamma_{r}=\gamma_{s}=0 \Longrightarrow s_{d f e}=1$, we recover the SIRFA example, for which the sharp threshold property holds ([50], (4.1)) that is, the disease-free Equilibrium is globally stable if $\mathcal{R}=\mathcal{R} s_{\text {dfe }} \leq 1$.

The endemic point simplifies to

$$
X^{(E E)}=\left(\frac{1}{\mathcal{R}}, \frac{\Lambda}{\beta}(\mathcal{R}-1), \frac{\gamma}{\beta}(\mathcal{R}-1)\right) .
$$

We may observe that the endemic point $X^{(E E)}$ is positive if $\mathcal{R}>1$. See Figure 6.

\section{Conclusions}

In this study, we completely analyzed the stability of a SIR model with eight parameters. This simple example began as an attempt to understand more complicated models, and the insights we obtained here were used [40]. We are currently unable to provide a complete stability analysis of analog SEIR models with many parameters, but we expect 
that limit cycles will intervene as well, by Smale's result [51] for Lotka Volterra models, which are similar to the epidemic models in the Ref. [40].

Author Contributions: F.A.; writing-review and editing, R.A.; writing-review and editing, G.B.; writing - review and editing, A.H.; writing—review and editing. All authors have read and agreed to the published version of the manuscript.

Funding: This research received no external funding.

Institutional Review Board Statement: Not applicable.

Informed Consent Statement: Not applicable.

Data Availability Statement: Not applicable.

Acknowledgments: We thank N. Bacaer for providing the references [7,52], and we thank the referees for their thorough reviews and suggestions.

Conflicts of Interest: The authors declare no conflict of interest.

\section{Appendix A. Auxiliary Lemmas}

Lemma A1. If only strict inequalities between $\Lambda+\gamma+v_{i}, \beta_{r}$ and $\beta$ are allowed, then the following 10 cases

$$
\left\{\begin{array}{l}
\Lambda+\gamma+v_{i}<\beta_{r}<\beta \\
\Lambda+\gamma+v_{i}<\beta<\beta_{r} \\
\beta_{r}<\Lambda+\gamma+v_{i}<\beta, \gamma_{s}<\gamma_{s}^{*} \\
\beta<\Lambda+\gamma+v_{i}<\beta_{r}, \gamma_{s} \geq \gamma_{s}^{*} \\
\beta_{r}<\Lambda+\gamma+v_{i}<\beta, v_{i}<\beta \\
\beta<v_{i}<\Lambda+\gamma+v_{i}<\beta_{r} \\
v_{i}<\beta<\Lambda+\gamma+v_{i}<\beta_{r}, \gamma_{s}<\gamma_{s}^{*}, \Delta<0 \\
v_{i}<\beta<\Lambda+\gamma+v_{i}<\beta_{r}, \gamma_{s}<\gamma_{s}^{*}, \Delta \geq 0 \\
\beta<\beta_{r}<\Lambda+\gamma+v_{i} \\
\beta_{r}<\beta<\Lambda+\gamma+v_{i}
\end{array}\right.
$$

form a disjoint decomposition of the parameter space.

Proof. We first note that in all cases where equality is allowed, the equality case may be arbitrarily assigned to any of the two cases it separates. It is therefore enough to only consider strict inequalities in this Lemma.

We want to show that the union of the 10 cases equals to the union of the six cases representing the possible orders of $\beta, \beta_{r}, \Lambda+\gamma+v_{i}$, which are

$$
\left\{\begin{array}{ll}
\beta>\beta_{r}>\gamma+v_{i}+\Lambda & 1 \\
\beta_{r}>\beta>\gamma+v_{i}+\Lambda & 2 \\
\beta>\gamma+v_{i}+\Lambda>\beta_{r} & 3 \\
\beta_{r}>\gamma+v_{i}+\Lambda>\beta & 4 \\
\gamma+v_{i}+\Lambda>\beta_{r}>\beta & 5 \\
\gamma+v_{i}+\Lambda>\beta>\beta_{r} & 6
\end{array} .\right.
$$

Now the cases 1,2 , with $\Lambda+\gamma+v_{i}<\min \left[\beta, \beta_{r}\right]$, and the cases 9,10 , with $\Lambda+\gamma+v_{i}>$ $\min \left[\beta, \beta_{e}\right]$ appear only once in the 10 cases of Theorem 1 , as case $1(a, b)$, and $2(d)(i, i i)$.

Next, we may check that the union of cases 3 and 5 (1(c) and 2(a) in the Theorem) together form permutation 3 . This requires checking the other two of the four formal cases, taking into account that the possible orders between $\beta, v_{i}$ and $\gamma_{s}, \gamma_{s}^{*}$ are void; the tedious verification is included in the Mathematica file available on our website. 
To conclude, it must be checked that the cases 4 and 6-8 (i.e., $1(\mathrm{~d}), 2(\mathrm{~b}), 2(\mathrm{c})(\mathrm{i}, \mathrm{ii})$ in the Theorem) together form a partition of the permutation 4 . First, note that cases 7 and 8 may be combined in $v_{i}<\beta<\Lambda+\gamma+v_{i}<\beta_{r}, \gamma_{s}<\gamma_{s}^{*}$. Next, we show in the Mathematica file that case $4 \beta<\Lambda+\gamma+v_{i}<\beta_{r}, \gamma_{s} \geq \gamma_{s}^{*}$ is incompatible with $\beta<v_{i}$, and so we can modify case 4 to $v_{i}<\beta<\Lambda+\gamma+v_{i}<\beta_{r}, \gamma_{s} \geq \gamma_{s}^{*}$. Thus, 4 and 6-8 become

$$
\left\{\begin{array}{l}
v_{i}<\beta<\Lambda+\gamma+v_{i}<\beta_{r}, \gamma_{s} \geq \gamma_{s}^{*} \\
\beta<v_{i}<\Lambda+\gamma+v_{i}<\beta_{r} \\
v_{i}<\beta<\Lambda+\gamma+v_{i}<\beta_{r}, \gamma_{s}<\gamma_{s}^{*},
\end{array}\right.
$$

whose union is clearly permutation 4 .

Lemma A2. (A) A necessary and sufficient condition for having precisely one endemic point with $s \in(0,1)$ is $C(A+B+C)<0$, where $A, B, C$ are defined in (29).

(B) Necessary and sufficient conditions for having precisely two endemic points with $s \in(0,1)$ are $\Delta>0$ and

$$
-2<\frac{B}{A}<0<\frac{C}{A}, \frac{B}{A}>-1-\frac{C}{A} .
$$

Proof. The conditions for having two roots bigger than 0 are $\frac{B}{A}<0<\frac{C}{A}$, and the conditions for having two roots smaller than 1 are obtained by applying these, after substituting $y=1-x$, yielding the result.

Since expressing these simple conditions in terms of the parameters of the model turned out to be quite difficult, we did not finalize this approach.

\section{References}

1. Anderson, R.M.; Anderson, B.; May, R.M. Infectious Diseases of Humans: Dynamics and Control; Oxford University Press: Oxford, UK, 1992.

2. Smith, H.L.; Thieme, H.R. Dynamical Systems and Population Persistence; American Mathematical Soc.: Providence, RI, USA, 2011; Volume 118.

3. Martcheva, M. An Introduction to Mathematical Epidemiology; Springer: New York, NY, USA, 2015; Volume 61.

4. Thieme, H.R. Mathematics in Population Biology; Princeton University Press: Princeton, NJ, USA, 2018.

5. Brauer, F.; Castillo-Chavez, C.; Feng, Z. Mathematical Models in Epidemiology; Springer: New York, NY, USA, 2019.

6. Mondaini, R.P. Trends in Biomathematics: Modeling Cells, Flows, Epidemics, and the Environment; Springer: Berlin/Heidelberg, Germany, 2020.

7. Bacaër, N. Mathématiques et épidémies, Cassini. 2021. pp. 1-320. Available online: https://store.cassini.fr/fr/enseignementdes-mathematiques/117-mathematiques-et-epidemie.html (accessed on 8 May 2012).

8. Della Marca, R. Problemi di Controllo in Epidemiologia Matematica e Comportamentale; Università degli studi di Modena e Reggio Emilia: Modena, Italy, 2021; pp. 1-163.

9. Kermack, W.O.; McKendrick, A.G. A contribution to the mathematical theory of epidemics. Proc. R. Soc. Lond. Ser. A Contain. Pap. A Math. Phys. Character 1927, 115, 700-721.

10. Earn, D.J. A light introduction to modelling recurrent epidemics. In Mathematical Epidemiology; Springer: Berlin/Heidelberg, Germany, 2008; pp. 3-17.

11. Schaback, R. On COVID-19 modelling. Jahresber. Der Dtsch. Math.-Ver. 2020, 122, 167-205. [CrossRef]

12. Bacaër, N. Un modèle mathématique des débuts de l'épidémie de coronavirus en France. Math. Model. Nat. Phenom. 2020, 15, 29. [CrossRef]

13. Ketcheson, D.I. Optimal control of an SIR epidemic through finite-time non-pharmaceutical intervention. arXiv 2020, arXiv:2004.08848.

14. Charpentier, A.; Elie, R.; Laurière, M.; Tran, V.C. COVID-19 pandemic control: Balancing detection policy and lockdown intervention under ICU sustainability. Math. Model. Nat. Phenom. 2020, 15, 57. [CrossRef]

15. Djidjou-Demasse, R.; Michalakis, Y.; Choisy, M.; Sofonea, M.T.; Alizon, S. Optimal COVID-19 epidemic control until vaccine deployment. medRxiv 2020,1-21. [CrossRef]

16. Sofonea, M.T.; Reyné, B.; Elie, B.; Djidjou-Demasse, R.; Selinger, C.; Michalakis, Y.; Alizon, S. Epidemiological Monitoring and Control Perspectives: Application of a Parsimonious Modelling Framework to the COVID-19 Dynamics in France. 2020; pp. 1-63. Available online: https://hal.archives-ouvertes.fr/hal-02619546/ (accessed on 8 May 2012).

17. Alvarez, F.E.; Argente, D.; Lippi, F. A Simple Planning Problem for COVID-19 Lockdown; Technical Report; National Bureau of Economic Research: Cambridge, MA, USA, 2020. 
18. Horstmeyer, L.; Kuehn, C.; Thurner, S. Balancing quarantine and self-distancing measures in adaptive epidemic networks. arXiv 2020, arXiv:2010.10516.

19. Di Lauro, F.; Kiss, I.Z.; Miller, J. Optimal timing of one-shot interventions for epidemic control. medRxiv 2020, 1-29. [CrossRef]

20. Franco, E. A feedback SIR (fSIR) model highlights advantages and limitations of infection-based social distancing. arXiv 2020, arXiv:2004.13216.

21. Baker, R. Reactive Social distancing in a SIR model of epidemics such as COVID-19. arXiv 2020, arXiv:2003.08285.

22. Caulkins, J.; Grass, D.; Feichtinger, G.; Hartl, R.; Kort, P.M.; Prskawetz, A.; Seidl, A.; Wrzaczek, S. How long should the COVID-19 lockdown continue? PLoS ONE 2020, 15, e0243413. [CrossRef] [PubMed]

23. Caulkins, J.P.; Grass, D.; Feichtinger, G.; Hartl, R.F.; Kort, P.M.; Prskawetz, A.; Seidl, A.; Wrzaczek, S. The optimal lockdown intensity for COVID-19. J. Math. Econ. 2021, 93, 102489. [CrossRef] [PubMed]

24. Kamgang, J.C.; Sallet, G. Computation of threshold conditions for epidemiological models and global stability of the disease-free equilibrium (DFE). Math. Biosci. 2008, 213, 1-12. [CrossRef] [PubMed]

25. Kaper, H.G.; Kaper, T.J. Asymptotic analysis of two reduction methods for systems of chemical reactions. Phys. D Nonlinear Phenom. 2002, 165, 66-93. [CrossRef]

26. Jardón-Kojakhmetov, H.; Kuehn, C.; Pugliese, A.; Sensi, M. A geometric analysis of the SIR, SIRS and SIRWS epidemiological models. Nonlinear Anal. Real World Appl. 2021, 58, 103220. [CrossRef]

27. Ginoux, J.M. Slow Invariant Manifolds of Slow-Fast Dynamical Systems. Int. J. Bifurc. Chaos 2021, 31, 2150112. [CrossRef]

28. de Jong, M.C.; Diekmann, O.; Heesterbeek, H. How does transmission of infection depend on population size. Epidemic Model. Their Struct. Relat. Data 1995, 84, 1-11.

29. De la Sen, M.; Nistal, R.; Alonso-Quesada, S.; Ibeas, A. Some formal results on positivity, stability, and endemic steady-state attainability based on linear algebraic tools for a class of epidemic models with eventual incommensurate delays. Discret. Dyn. Nat. Soc. 2019, 2019, 22. [CrossRef]

30. Busenberg, S.; van den Driessche, P. Analysis of a disease transmission model in a population with varying size. J. Math. Biol. 1990, 28, 257-270. [CrossRef]

31. Busenberg, S.; van den Driessche, P. A method for proving the non-existence of limit cycles. J. Math. Anal. Appl. 1993, 172, 463-479. [CrossRef]

32. Derrick, W.; van den Driessche, P. A disease transmission model in a nonconstant population. J. Math. Biol. 1993, 31, 495-512. [CrossRef] [PubMed]

33. Greenhalgh, D. Hopf bifurcation in epidemic models with a latent period and nonpermanent immunity. Math. Comput. Model. 1997, 25, 85-107. [CrossRef]

34. Li, M.Y.; Graef, J.R.; Wang, L.; Karsai, J. Global dynamics of a SEIR model with varying total population size. Math. Biosci. 1999, 160, 191-213. [CrossRef]

35. Razvan, M. Multiple equilibria for an SIRS epidemiological system. arXiv 2001, arXiv:0101051.

36. Li, J.; Ma, Z. Qualitative analyses of SIS epidemic model with vaccination and varying total population size. Math. Comput. Model. 2002, 35, 1235-1243. [CrossRef]

37. Sun, C.; Hsieh, Y.H. Global analysis of an SEIR model with varying population size and vaccination. Appl. Math. Model. 2010, 34, 2685-2697. [CrossRef]

38. Yang, W.; Sun, C.; Arino, J. Global analysis for a general epidemiological model with vaccination and varying population. J. Math. Anal. Appl. 2010, 372, 208-223. [CrossRef]

39. Lu, G.; Lu, Z. Global asymptotic stability for the SEIRS models with varying total population size. Math. Biosci. 2018, 296, 17-25. [CrossRef]

40. Avram, F.; Adenane, R.; Basnarkov, L.; Bianchin, G.; Goreac, D.; Halanay, A. On matrix-SIR Arino models with linear birth rate, loss of immunity, disease and vaccination fatalities, and their approximations. arXiv 2021, arXiv:2112.03436.

41. Van den Driessche, P.; Watmough, J. Further Notes on the Basic Reproduction Number. In Mathematical Epidemiology; Springer: Berlin/Heidelberg, Germany, 2008; pp. 159-178.

42. Rashkov, P. A model for a vector-borne disease with control based on mosquito repellents: A viability analysis. J. Math. Anal. Appl. 2021, 498, 124958. [CrossRef]

43. Hethcote, H.W. Qualitative analyses of communicable disease models. Math. Biosci. 1976, 28, 335-356. [CrossRef]

44. Miclo, L.; Spiro, D.; Weibull, J. Optimal epidemic suppression under an ICU constraint. arXiv 2020, arXiv:2005.01327.

45. Avram, F.; Freddi, L.; Goreac, D. Optimal control of a SIR epidemic with ICU constraints and target objectives. Appl. Math. Comput. 2022, 418, 126816. [CrossRef]

46. van den Driessche, P.; Watmough, J. Reproduction numbers and sub-threshold endemic equilibria for compartmental models of disease transmission. Math. Biosci. 2002, 180, 29-48. [CrossRef]

47. Hirsch, M.; Smale, D. Differential Equations, Dynamical Systems and Linear Algebra; Academic-Press: Cambridge, MA, USA, 1974.

48. Mena-Lorcat, J.; Hethcote, H.W. Dynamic models of infectious diseases as regulators of population sizes. J. Math. Biol. 1992, 30, 693-716. [CrossRef]

49. Vargas-De-León, C. Constructions of Lyapunov functions for classic SIS, SIR and SIRS epidemic models with variable population size. Foro-Red Rev. Electrónica De Conten. Matemático 2009, 26, 1-12. 
50. Shuai, Z.; van den Driessche, P. Global stability of infectious disease models using Lyapunov functions. SIAM J. Appl. Math. 2013, 73, 1513-1532. [CrossRef]

51. Smale, S. On the differential equations of species in competition. J. Math. Biol. 1976, 3, 5-7. [CrossRef]

52. Lotka, A.J. Analyse Démographique Avec Application Particulière à l'espèce Humaine; Hermann: Paris, France, 1939. 